\title{
A wave driver theory for vortical waves propagating across junctions with application to those between rigid and compliant walls
}

\author{
P. K. SEN ${ }^{1} \dagger$, P. W. CARPENTER ${ }^{2} \ddagger$, S. HEGDE \\ AND C. D A VIES $\mathbf{S}^{3}$ \\ ${ }^{1}$ Department of Applied Mechanics, Indian Institute of Technology, New Delhi 110016, India \\ ${ }^{2}$ School of Engineering, University of Warwick, Coventry CV4 7AL, UK \\ ${ }^{3}$ School of Mathematics, Cardiff University, Cardiff CF24 4YH, UK
}

(Received 31 July 2007 and in revised form 19 November 2008)

A theory is described for propagation of vortical waves across alternate rigid and compliant panels. The structure in the fluid side at the junction of panels is a highly vortical narrow viscous structure which is idealized as a wave driver. The wave driver is modelled as a 'half source cum half sink'. The incoming wave terminates into this structure and the outgoing wave emanates from it. The model is described by half Fourier-Laplace transforms respectively for the upstream and downstream sides of the junction. The cases below cutoff and above cutoff frequencies are studied. The theory completely reproduces the direct numerical simulation results of Davies \& Carpenter (J. Fluid Mech., vol. 335, 1997, p. 361). Particularly, the jumps across the junction in the kinetic energy integral, the vorticity integral and other related quantities as obtained in the work of Davies \& Carpenter are completely reproduced. Also, some important new concepts emerge, notable amongst which is the concept of the pseudo group velocity.

\section{Introduction}

Waves incident on a junction between two different wave-bearing media are commonly met in many practical applications. In many cases well-established techniques exist for calculating the transmitted, reflected and diffracted waves in terms of the incident wave at the junction (Lighthill 1978; Billingham \& King 2000). But no suitable method appears to have been developed for calculating the amplitude of the transmitted wave when a rotational (vortical) wave in a fluid is incident on a junction with a different wave-bearing system. Here we present a novel method for solving such problems.

We believe that aspects of our method are generic, but it is presented in the context of a specific problem that is illustrated in figure 1. This depicts a Tollmien-Schlichting (TS) wave incident on a junction at $x=\ell_{1}$ between a plane channel flow with rigid and compliant walls. The compliant section of the channel is finite in length, and so there is a second junction at $x=\ell_{2}$ between the compliant and rigid sections. This problem was investigated by Davies \& Carpenter (1997) using direct numerical simulations. 


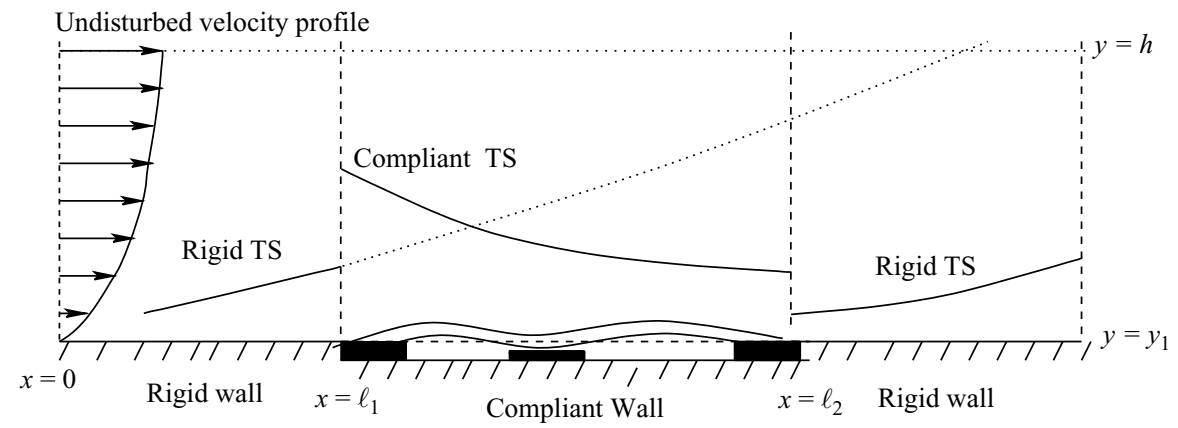

FIGURE 1. Schematic sketch of the problem under study. The figure is taken from figure 2 of Davies \& Carpenter (1997).

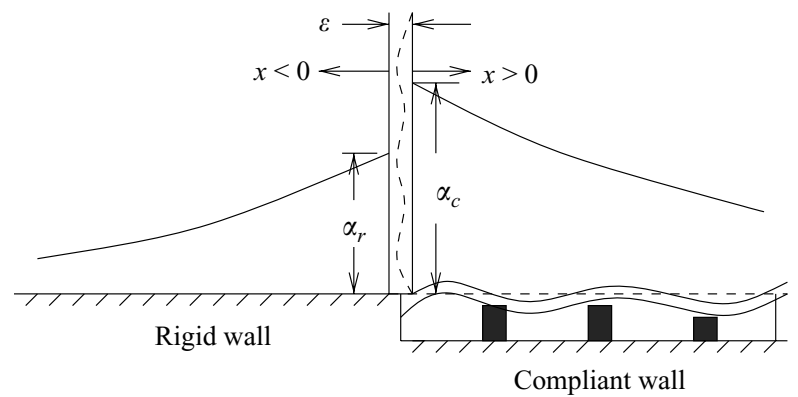

FIGURE 2. Schematic sketch defining the notation used to analyse the behaviour of the incident and transmitted waves in the neighbourhood of the junction. The broken sinuous curve depicted within the narrow junction zone is intended to depict the wave driver created by the interaction of the incident wave and the junction.

Although the numerical simulations provided a satisfactory solution to the problem, they exhibited certain puzzling features that could not be explained. One of these concerned the spatial development of the perturbation enstrophy and kinetic energy integrals across the two junctions. When the incident wave is below the cutoff frequency of the compliant wall, the only propagating mode is the TS wave (see schematic in figure 2). It is immediately evident from figure 6 of Davies \& Carpenter (1997) that in this case there are sharp jumps at the leading-edge junction in the enstrophy and kinetic energy integrals, downwards for the former, upwards for the latter and vice versa at the trailing-edge junction. All simulations with the TS frequency below the cutoff frequency of the compliant wall displayed the same behaviour. Accordingly, the questions remaining unanswered by the numerical simulations are as follows: Why do the jumps occur and why is there a kind of reciprocity relationship between the leading- and trailing-edge junctions?

We now give a brief description of our novel analytical approach to this problem. For the present, to avoid unnecessary complication we shall consider the leading edge of the compliant panel to be the sole junction. Thus we write the perturbation streamfunction in the following form:

$$
\psi(x, y, t)=f\left(x^{\prime}=x-\ell_{1}, y\right) \mathrm{e}^{-\mathrm{i} \omega t},
$$

where $\bar{\omega}$ is the disturbance frequency, $x$ and $y$ are respectively the streamwise and wall-normal coordinates and $t$ the time; the origin of $x^{\prime}$ coincides with the junction. 
We expect the streamfunction to take different forms upstream and downstream of the junction, so we write

$$
f\left(x^{\prime}, y\right)=H\left(-x^{\prime}\right) f_{r}\left(x^{\prime}, y\right)+H\left(x^{\prime}\right) f_{c}\left(x^{\prime}, y\right),
$$

where $H\left(x^{\prime}\right)$ is the Heaviside step function and suffices $r$ and $c$ denote rigid and compliant walls, respectively. Also plotted in figure 6 of Davies \& Carpenter (1997) are the envelopes corresponding to the growing TS wave over an infinitely long rigid wall and the attenuating TS wave over an infinitely long compliant wall. These were obtained by solving the coupled Orr-Sommerfeld compliant-wall eigenproblems for the corresponding homogeneous systems. It is evident that only in the immediate vicinity of the junction do the growth rates of the incident and transmitted waves depart from those corresponding to the (homogeneous) eigenproblems. This suggests that the junction region is very narrow compared with the wavelength of the incident TS wave (see figure 2). If this is so, the functions in (1.2) can be written in the forms

$$
\begin{aligned}
& f_{r}\left(x^{\prime}, y\right)=\operatorname{Re}[\underbrace{a \bar{\phi}_{r}(y) \mathrm{e}^{\mathrm{i} \bar{\alpha}_{r} x^{\prime}}}_{\text {far field }}+\underbrace{f_{n r}(\xi, y)}_{\text {near field }}]: x^{\prime}<0, \\
& f_{c}\left(x^{\prime}, y\right)=\operatorname{Re}[\underbrace{\lambda a \bar{\phi}_{c}(y) \mathrm{e}^{\mathrm{i} \bar{\alpha}_{c} x^{\prime}}}_{\text {far field }}+\underbrace{f_{n c}(\xi, y)}_{\text {near field }}]: \quad x^{\prime}>0,
\end{aligned}
$$

where Re indicates that the real part of the complex quantities is taken, $a$ is the amplitude of the incident TS wave at $x^{\prime}=0$ (hereafter taken as unity for the leadingedge junction) and $\xi=x^{\prime} / \epsilon ; \epsilon$ is assumed to be a measure of the non-dimensional width of the junction region. There is no loss of generality in the forms assumed in $(1.3 a, b)$. However, they will only be useful if $\epsilon=O(1)$. In fact, it will be shown later to be $O\left(R e^{-1 / 3}\right)$ where $R e$ is the Reynolds number. The far-field eigenfunctions $\bar{\phi}_{r}$ and $\bar{\phi}_{c}$ and eigenvalues $\bar{\alpha}_{r}$ and $\bar{\alpha}_{c}$, upstream (rigid) and downstream (compliant) of the junction, correspond to suitably normalized eigenmodes of the respective homogeneous systems, and $\lambda$ is an unknown constant representing the amplitude ratio of the downstream to upstream far-field solutions. Assuming that the homogeneous eigenmodes are known, the problem we set out to solve is how to determine the amplitude ratio $\lambda$ without detailed knowledge of the near field.

By using half-range Fourier-Laplace transforms (HFLTs) of the governing equation, we show that the junction is equivalent to a virtual local wave driver. This concept is illustrated in figure 2. The problem then reduces to determining the strength of the wave driver. We use the classic theory for inhomogeneous ordinary differential equations, which makes use of the adjoint eigenfunctions, to determine the strength of the virtual driver and the amplitude ratio $\lambda$. It is known that near-field effects can be simulated exactly by a virtual wave driver for some simple problems. For example, Carpenter et al. (2002) and Carpenter \& Sen (2003) show that this is so for elastic waves propagating along an elastic plate-spring system and incident on a pinned joint with another elastic plate-spring system.

In some respects Hill (1995) could be regarded as the main precursor to our approach (we were shamefully unaware of his excellent paper when we wrote our earlier papers). He showed how the adjoint TS eigensolution could be interpreted physically and mathematically as indicating the amplitude of the unstable disturbance that results from periodic forcing by a vorticity source. However, our problem differs from conventional receptivity problems in a crucial respect; in our case the strength of the vorticity source (or driver) is unknown a priori and has to be determined as 
part of the theory. Accordingly, in our theory the adjoint eigensolution plays a sort of reverse role compared with Hill, in that it determines the strength of the vorticity source (or driver) created at the junction by the incident TS wave.

Before presenting our wave driver theory in detail, it may be worthwhile to discuss briefly some other approaches to similar problems that do not work for vortical waves. Many problems involving waves incident on junctions can be solved by using conservation relations. A well-known example is waves along elastic tubes (Lighthill 1978). In this case the reflected and transmitted waves at a junction can be determined in terms of the incident wave by applying continuity of pressure and either conservation of mass or energy; these last two conditions lead to identical results. An example that is similar to the present problem and can be solved by conservation methods concerns waves propagating in a potential flow along a compliant wall with spatially varying stiffness (see Lucey, Sen \& Carpenter 2003). At first sight it might appear that conservation of properties such as energy and enstrophy could be used to solve the present problem. It is immediately apparent from figure 6 of Davies \& Carpenter (1997), however, that neither quantity is conserved across the junctions. As pointed out above there are jumps in the values of both quantities at the junctions. From the viewpoint of our theory, this is because the near field at the junction, of which the virtual wave driver is an idealization, creates positive, or negative, energy and enstrophy at the junctions.

Another approach that could appear viable is to confine attention to the flow regime downstream of the junction (i.e. over the compliant wall) and to take the upstream incident eigenstate as the initial condition. One might attempt to expand this initial condition in terms of a set of downstream eigenmodes. However, this approach will fail because although the eigenmodes for the two-dimensional OrrSommerfeld eigensystem for the rigid-wall channel are bi-orthogonal (Henningson \& Schmid 1992), as also are the corresponding ones for the Orr-Sommerfeld compliantwall eigensystem, neither set of eigenfunctions is complete and it is not possible to describe the compliant-wall eigenfunctions in terms of expansions of the rigid-wall ones or vice versa. In simple terms, the different forms of the wall boundary conditions plainly make it impossible, as can be seen from the forms of the eigenfunctions and their adjoints. Even if such an approach were feasible, it would still be invalid because the near field is completely neglected. That neglecting the near field in this way leads to errors is illustrated by comparing the results of Wiplier \& Ehrenstein (2000) and Davies \& Carpenter (1997). They adopted an approach whereby the incident TS wave was used as the initial condition at the junction for their direct numerical simulations. The flow fields downstream of the junction produced by their simulations differ significantly from the corresponding simulations obtained using the methods of Davies and Carpenter. This is not surprising because, in effect, the near field upstream of the junction is being ignored. (However, we are not suggesting that this in any way invalidates the study of Wiplier \& Ehrenstein.)

It has sometimes been suggested to us in discussions with others that the WeinerHopf technique (Noble 1958, Billingham \& King 2000) would be a suitable approach. The applications of the Wiener-Hopf technique known to us involve irrotational waves, primarily acoustic waves. As with our approach, half-range Fourier transforms are used. The classic problem presented in Billingham \& King (2000) is the diffraction of acoustic waves by a semi-infinite plate extending from $x=0$ to $x=\infty$. Somewhat analogous to (1.2) the Fourier transform of the perturbation potential takes different forms for $y>0$ and $y<0$. The Weiner-Hopf technique makes use of the fact that the two forms have an overlapping region of analyticity. It then introduces and uses 


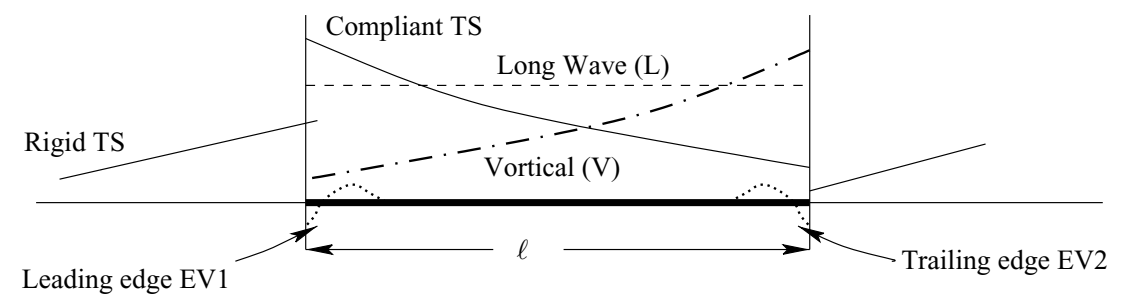

FIGURE 3. Schematic sketch of the eigenmodes for the case above cutoff frequency of the compliant panel. There are three propagating modes present over the compliant panel. The transmitted TS wave which is damped, a near-neutral almost irrotational damped long-wall wave (L) that propagates downstream from the leading-edge junction and a vortical damped-wall wave (V) that propagates upstream from the trailing-edge junction. There are also evanescent modes (EV1 and EV2) in the vicinity of the junctions.

integrals with respect to $x$ of the jump in the Fourier transform of the potential at $y=0$ and its normal derivatives over positive and negative half-infinite ranges. One of the integrals is zero and the boundary conditions at $y=0$ and the form assumed for the transformed potential allow relationships to be derived between the remaining integrals. Solving these leads to a solution of the problem. This is typical of the application of the Weiner-Hopf technique. Our method is plainly quite different. Moreover, although we could not entirely rule out the Weiner-Hopf technique as a possible approach, we could see no way of applying it to our problem. A major difference is that the Weiner-Hopf technique leads to the exact solution of a problem, whereas our method seeks to determine the relationship between the far-field solutions without recourse to a detailed knowledge of the near field.

Ours is essentially a receptivity problem. Some specialists in receptivity have made unpublished attempts to solve it using receptivity techniques. For example, Manuilovich (2001), drawing on earlier work (Manuilovich 1992), conceives of the junction as being analogous to a controlled wall motion that creates a TS wave. This is evidently somewhat similar to a wave driver. However, Manuilovich does not actually solve the present problem, originating with Davies \& Carpenter (1997). A brief description of his approach is given by Carpenter \& Sen (2003). However, in more recent papers (Manuilovich 2003, 2004a, b) the present problem and other similar ones have been investigated.

When the frequency of incident TS wave is above the cutoff frequency of the compliant wall, there are three propagating waves plus evanescent modes (EV1 and EV2) over the compliant wall (see figure 3). As well as the TS wave, there is an attenuating vortical wall mode $(\mathrm{V})$ propagating in the upstream direction from the trailing-edge junction and a long-wave near-neutral almost irrotational (L) mode propagating downstream from the leading-edge junction. It was shown in Davies $\&$ Carpenter (1997) that they are all eigenmodes of the coupled Orr-Sommerfeld compliant-wall eigenproblem. In some numerical simulations for long compliant panels using the methods of Davies \& Carpenter (1997), we found that even when the TS wave was virtually reduced to zero amplitude over the compliant wall, the transmitted TS wave propagating downstream from the trailing-edge junction often had a substantial amplitude. This puzzling feature was not reported in Davies \& Carpenter (1997). It is shown below that the damped vortical wall mode propagating upstream from the trailing-edge junction actually creates a virtual wave driver and this generates the downstream-propagating TS wave. In cases where the TS wave has 
not been completely suppressed by the time it reaches the trailing-edge junction, both it and the vortical wall mode create separate virtual wave drivers at the junction.

The study in Davies \& Carpenter (1997) was mainly motivated by an interest in using a series of short compliant panels to suppress the growth of TS waves, thereby maintaining laminar flow at very high Reynolds numbers. But our problem is plainly related to many biomedical applications; for example, the use of stents to reinforce blood vessels, and pulsatile flows through a stenosis. There are also evident links with the study of collapsible tubes (see for example Heil \& Jensen 2003; Grotberg \& Jensen 2004), particularly the numerical studies of Luo \& Pedley $(1995,1996)$ and Pedrizzetti (1998). However, these studies are nonlinear, whereas the general problem considered here is linear. In other branches of engineering, similar problems are found in a variety of applications (see for example Nguyen, Païdoussis \& Misra 1994; Howe 1998; Païdoussis 1998).

The remainder of the paper is set out as follows. The formulation of the problem is described in detail in $\S 2$. The development of the theory based on modelling the junction as a local virtual wave driver, for the rigid-wall case, is discussed in $\S 3$. This problem is extended to the compliant cases in $\S 4$. Section 5 discusses the form of the wave driver function. The results are presented and discussed in $\S 6$. Finally the conclusions are given in $\S 7$.

\section{Formulation of the governing equations}

\subsection{Statement of the fundamental problem}

As mentioned earlier, in the present work a channel (plane Poiseuille) flow is considered as the model wave-bearing medium for demonstrating our novel approach to waves propagating across junctions. Also the numerical simulations of Davies \& Carpenter (1997) are available for comparison. Furthermore, plane Poiseuille flow is a good choice for the model problem because it is a truly parallel flow with a simple parabolic velocity profile. As shown in figure 1, the rigid-compliant-rigid channel region is formed by having a compliant insert between two rigid sections. The streamwise and wall-normal coordinates are denoted by $x$ and $y$ respectively. The compliant wall properties are applicable in the region from $x=\ell_{1}$ to $x=\ell_{2}$ as shown in figure 1. The regions $x<\ell_{1}$ and $x>\ell_{2}$ correspond to the rigid-wall channel sections. The domain from $y=0$ to $y=h$ corresponds to the half-width of the channel. The reference length, velocity and time scales used for non-dimensionalization are $h, U_{m}$ and $h / U_{m}$ respectively, where $U_{m}$ is the centreline undisturbed velocity. Henceforth the variables and parameters are dimensionless unless explicitly indicated otherwise. The Reynolds number is given as $R e=U_{m} h / v$, where $v$ is the kinematic viscosity.

Since a two-dimensional parallel flow is being considered, a disturbance streamfunction $\psi(x, y, t)$ can be introduced. The amplitudes of the disturbances are assumed to be sufficiently small for linearized theory to be used throughout. The nondimensional governing equation, obtained by linearizing the Navier-Stokes equations, is written below in vorticity $\left(\nabla^{2} \psi\right)$ form in terms of the disturbance streamfunction:

$$
\frac{\partial}{\partial t}\left(\nabla^{2} \psi\right)+\bar{u} \frac{\partial}{\partial x}\left(\nabla^{2} \psi\right)-\bar{u}^{\prime \prime} \frac{\partial \psi}{\partial x}-\frac{1}{R e} \nabla^{4} \psi=0,
$$

where $\bar{u}$ is the undisturbed laminar velocity profile given as $\bar{u}=2 y-y^{2}$.

The TS wave incident on the leading-edge junction is assumed to be monochromatic with frequency $\bar{\omega}$, and so $\psi$ takes the form given in (1.1). We shall develop the theory for the case of a TS wave propagating along the inlet rigid section of the channel 
and incident on the junction with the compliant section. We consider initially the case when $\bar{\omega}$ is below the cutoff frequency of the compliant wall. Thus the only wave propagating along the compliant section will be the transmitted TS wave. Under these circumstances the streamfunction can take the form of (1.1) and substituting this into (2.1) gives the operative differential equation for the problem, namely

$$
-\mathrm{i} \bar{\omega}\left(\nabla^{2} f\right)+\bar{u} \frac{\partial}{\partial x}\left(\nabla^{2} f\right)-\bar{u}^{\prime \prime} \frac{\partial f}{\partial x}-\frac{1}{R e} \nabla^{4} f=0 .
$$

Following Davies \& Carpenter (1997) we restrict attention to normal disturbance velocity components $v$ that are symmetrical (implying antisymmetrical streamwise disturbance components $u$ and symmetrical disturbance streamfunction and vorticity), because disturbances of this type determine the stability in the case of rigid walls (Drazin \& Reid 1981). Thus for the rigid- and compliant-wall sections alike of the channel, the boundary conditions at the centreline are

$$
f_{y}(x, 1)=f_{y y y}(x, 1)=0,
$$

where suffices $y$ and $x$ denote partial differentiation with respect to these variables.

On the rigid wall the usual no-penetration and no-slip conditions hold, i.e.

$$
f_{x}(x, 0)=f_{y}(x, 0)=0 .
$$

On the compliant wall these, when linearized about $y=0$, take the form (see for example Carpenter \& Garrad 1985)

$$
f_{x}(x, 0)=\mathrm{i} \bar{\omega} \hat{\eta}, \quad f_{y}(x, 0)+\bar{u}^{\prime}(0) \hat{\eta}=0,
$$

where $\eta=\hat{\eta} \exp (\mathrm{i} \bar{\omega} t)$ is the wall displacement. Equations $(2.5 a)$ and $(2.5 b)$ can be combined in a single boundary condition of the form

$$
f_{y}(x, 0)-\frac{\mathrm{i} \bar{u}^{\prime}(0)}{\bar{\omega}} f_{x}(x, 0)=0 .
$$

The remaining boundary condition at the wall is derived from the equation of motion for the compliant wall. Following Davies \& Carpenter (1997) we use the plate-spring compliant-wall model of Carpenter \& Garrad (1985). Accordingly the wall displacement $\eta$ is only in the $y$ direction and is governed by the following equation of motion:

$$
m \frac{\partial^{2} \eta}{\partial t^{2}}+d \frac{\partial \eta}{\partial t}+B \frac{\partial^{4} \eta}{\partial x^{4}}+K \eta=-p_{w},
$$

where $p_{w}$ is the unsteady hydrodynamic pressure acting on the wall and the nondimensional wall parameters are defined as follows in terms of dimensional quantities denoted by an asterisk:

$$
\begin{gathered}
m=\frac{m^{*}}{\rho h}, \quad \frac{d^{*}}{\rho U_{m}} \equiv \frac{1}{R e} \frac{d^{*} \rho h}{v} \equiv \frac{d}{R e}, \\
\frac{B^{*}}{\rho h^{3} U_{m}^{2}} \equiv \frac{1}{R e^{2}} \frac{B^{*}}{\rho h v^{2}} \equiv \frac{B}{R e^{2}}, \quad \frac{K^{*} h}{\rho U_{m}^{2}}=\frac{1}{R e^{2}} \frac{K^{*} h^{3}}{\rho v^{2}}=\frac{K}{R e^{2}},
\end{gathered}
$$

where $m^{*}, d^{*}, B^{*}$ and $K^{*}$ are respectively the mass per unit length, damping, flexural rigidity and spring stiffness of the plate-spring system.

If the plate is pinned at each junction,

$$
\eta=\frac{\partial^{2} \eta}{\partial x^{2}}=0 \quad \text { at } \quad x=\ell_{1}, \ell_{2},
$$


whereas for clamped end conditions

$$
\eta=\frac{\partial \eta}{\partial x}=0 \quad \text { at } \quad x=\ell_{1}, \ell_{2}
$$

Pinned joints were assumed in Davies \& Carpenter (1997), but there is no difficulty in carrying out numerical simulations for clamped end conditions, and some results for such end conditions will be presented below.

Two things are required to convert (2.7) into a boundary condition for the streamfunction $f$. First, we need to replace $\eta$ as the variable with $\hat{\eta}$, and then substitute for $\hat{\eta}$ using $(2.5 a)$. Second, we need a way of determining the wall pressure $p_{w}=\hat{p}_{w} \exp (-\mathrm{i} \bar{\omega} t)$. In Davies \& Carpenter (1997) we used the linearized $y$-momentum equation to determine $p_{w}$ as an integral with respect to $y$ across the channel. This is very convenient for our particular form of numerical simulation. In the present context, however, it has the disadvantage of leading to a non-standard form for the boundary condition that makes it difficult to apply the classic theory for inhomogeneous systems of ordinary differential equations (see for example Ince 1926). So, as an alternative, we follow Carpenter \& Garrad (1985) and use the linearized $x$-momentum equation that takes the form

$$
\hat{p}_{w x}+\frac{1}{R e}\left(f_{y x x}(x, 0)+f_{y y y}(x, 0)\right)=0 .
$$

Note that the inertial terms are omitted in (2.11) because they sum to zero at $y=0$ on account of the no-slip condition, as given in $(2.5 b)$.

In $\S 2.2$ below, the final forms of the boundary conditions are formulated after the HFLTs have been taken.

The two-dimensional TS wave incident on the leading-edge junction at $x=\ell_{1}$ undergoes a jump in amplitude, as discussed above. Another apparently nearreciprocal jump in amplitude occurs at the trailing-edge junction at $x=\ell_{2}$ (see figure 1 and Davies \& Carpenter 1997). One needs to understand the conditions at the junctions, $x=\ell_{1}$ and $x=\ell_{2}$, to understand why these jumps occur and to determine the transmitted wave and, in some applications, the reflected wave at each junction. Figure 2 also depicts schematically typical growth curves for the disturbance waves either side of the junctions. This behaviour will be explained in later sections.

\subsection{The Fourier-Laplace transformed system of equations}

Half-range Fourier-Laplace transforms (HFLTs) in terms of wavenumber $\alpha$ are now defined for $f(x, y)=f_{r}\left(x^{\prime}, y\right) H\left(-x^{\prime}\right)+f_{c}\left(x^{\prime}, y\right) H\left(x^{\prime}\right)$, again in two halves, for either side of the junction. (For simplicity we will work with a shifted streamwise variable $x^{\prime}=x-\ell_{1}$ that has its origin at the junction.)

$$
\begin{aligned}
& F_{r}(y, \alpha)=\int_{-\infty}^{0} f_{r}\left(x^{\prime}, y\right) \mathrm{e}^{-\mathrm{i} \alpha x^{\prime}} \mathrm{d} x^{\prime}, \\
& F_{c}(y, \alpha)=\int_{0}^{+\infty} f_{c}\left(x^{\prime}, y\right) \mathrm{e}^{-\mathrm{i} \alpha x^{\prime}} \mathrm{d} x^{\prime} .
\end{aligned}
$$

The form of the solutions must be such that away from the immediate vicinity of the junction they reduce to the far-field TS waves. Thus we expect them to take the forms given in $(1.3 a, b)$. The inverse transforms are also defined separately for $-\infty<x^{\prime} \leqslant 0$ and $0 \leqslant x^{\prime}<\infty$ and can be evaluated as follows:

$$
f_{r}\left(x^{\prime}, y\right)=\frac{1}{2 \pi} \int_{C_{r}} F_{r}(y, \alpha) \mathrm{e}^{\mathrm{i} \alpha x^{\prime}} \mathrm{d} \alpha, \quad-\infty<x^{\prime} \leqslant 0,
$$




$$
f_{c}\left(x^{\prime}, y\right)=\frac{1}{2 \pi} \int_{C_{c}} F_{c}(y, \alpha) \mathrm{e}^{\mathrm{i} \alpha x^{\prime}} \mathrm{d} \alpha, \quad 0 \leqslant x^{\prime}<\infty,
$$

where $C_{r}$ and $C_{c}$ are suitable contours enclosing the poles. The above method of representation is most convenient for showing that the junction can be modelled as a local virtual wave driver. This is demonstrated below.

First, it is important to note how derivatives with respect to $x$ are dealt with for HFLTs. Thus, using integration by parts, the HFLT of the first derivative of the stream functions, $f_{r, c}\left(x^{\prime}, y\right)$, upstream and downstream of the junction are given by

$$
\begin{aligned}
& \int_{-\infty}^{0} \frac{\partial f_{r}}{\partial x} \mathrm{e}^{-\mathrm{i} \alpha x^{\prime}} \mathrm{d} x^{\prime}=f_{r}(0, y)+\mathrm{i} \alpha F_{r}(y ; \alpha), \\
& \int_{0}^{\infty} \frac{\partial f_{c}}{\partial x} \mathrm{e}^{-\mathrm{i} \alpha x^{\prime}} \mathrm{d} x^{\prime}=-f_{c}(0, y)+\mathrm{i} \alpha F_{c}(y ; \alpha),
\end{aligned}
$$

using repeated integration by parts, the HFLTs of the corresponding higher derivatives are given by

$$
\begin{aligned}
& \int_{-\infty}^{0} \frac{\partial^{n} f_{r}}{\partial x^{n}} \mathrm{e}^{-\mathrm{i} \alpha x^{\prime}} \mathrm{d} x=\sum_{k=1}^{n}(\mathrm{i} \alpha)^{k-1} f_{r}^{(n-k)}(0, y)+(\mathrm{i} \alpha)^{n} F_{r}(y ; \alpha), \\
& \int_{0}^{\infty} \frac{\partial^{n} f_{c}}{\partial x^{n}} \mathrm{e}^{-\mathrm{i} \alpha x^{\prime}} \mathrm{d} x=-\sum_{k=1}^{n}(\mathrm{i} \alpha)^{k-1} f_{c}^{(n-k)}(0, y)+(\mathrm{i} \alpha)^{n} F_{c}(y ; \alpha) .
\end{aligned}
$$

Other variables are dealt with in a similar fashion.

Taking the HFLT of the operative differential equation (2.2), and making use of the results given in $(2.14 a, b)$ and $(2.15 a, b)$, we obtain the following pair of equations:

$$
\begin{aligned}
& L(\alpha) F_{r, c}= \pm \bar{\omega}\{\alpha f_{r, c}+\mathrm{i} \underbrace{\left(\frac{\partial f_{r, c}}{\partial x}\right)}_{O(1 / \epsilon)}\}_{x^{\prime}=0} \\
& \pm \bar{u}\{-\alpha^{2} f_{r, c}+\mathrm{i} \alpha \underbrace{\left(\frac{\partial f_{r, c}}{\partial x}\right)}_{O(1 / \epsilon)}+\underbrace{\left(\frac{\partial^{2} f_{r, c}}{\partial x^{2}}\right)}_{O\left(1 / \epsilon^{2}\right)}\}_{x^{\prime}=0} \mp \bar{u}^{\prime \prime}\left\{f_{r, c}\right\}_{x^{\prime}=0} \\
& \mp \frac{2}{R e}\{\mathrm{i} \alpha f_{r, c}^{\prime \prime}+\underbrace{\left(\frac{\partial f_{r, c}^{\prime \prime}}{\partial x}\right)}_{O(1 / \epsilon)}\}_{x^{\prime}=0} \\
& \mp \frac{1}{R e}\{-\mathrm{i} \alpha^{3} f_{r, c}-\alpha^{2} \underbrace{\left(\frac{\partial f_{r, c}}{\partial x}\right)}_{O(1 / \epsilon)}+\mathrm{i} \alpha \underbrace{\left(\frac{\partial^{2} f_{r, c}}{\partial x^{2}}\right)}_{O\left(1 / \epsilon^{2}\right)}+\underbrace{\left(\frac{\partial^{3} f_{r, c}}{\partial x^{3}}\right)}_{O(1 / \epsilon)^{3}}\}_{x^{\prime}=0}, \quad(2.16 a, b)
\end{aligned}
$$


where $L(\alpha)$ is the linear Orr-Sommerfeld operator, namely

$$
L(\alpha) F=\mathrm{i}(\alpha \bar{u}-\bar{\omega})\left(F^{\prime \prime}-\alpha^{2} F\right)-\mathrm{i} \alpha \bar{u}^{\prime \prime} F-\frac{1}{R e}\left(F^{\prime \prime \prime \prime}-2 \alpha^{2} F^{\prime \prime}+\alpha^{4} F\right) .
$$

In the above, (2.16a) and suffix $r$ correspond to $x^{\prime}<0$ whereas (2.16b) and suffix $c$ correspond to $x^{\prime}>0$. Also, when \pm and $\mp$ are used the upper symbol corresponds to $(2.16 a)$ and the lower to $(2.16 b)$. Also, assuming for now that the form proposed in $(1.3 a, b)$ is valid, the order of magnitude of each derivative of $f$ with respect to $x$, at $x^{\prime}=0$, is indicated below the respective terms. These follow from the forms assumed in $(1.3 a, b)$. Superscript prime denotes derivatives with respect to $y$.

As it is a solution of the Navier-Stokes equations, $f$ and all its derivatives are continuous everywhere within the fluid domain including at the junction. This does not, however, strictly apply at the point $\left(x^{\prime}=0, y^{\prime}=0\right)$ where, depending on the type of joint assumed between the rigid and compliant walls, any of the second and higher derivatives of $f$ could be discontinuous. For example, recalling from (2.5a) that $\eta \propto f_{x}\left(x^{\prime}, 0\right)$, for pinned joints (see $\left.(2.9 a, b)\right), f_{x}\left(x^{\prime}, 0\right)=f_{x x x}\left(x^{\prime}, 0\right)=0$ as $x^{\prime} \rightarrow 0^{+}$and so are continuous along the wall at the junction, but $f_{x x}\left(x^{\prime}, 0\right)$ is not. This minor difficulty arises because in the immediate vicinity of the pinned joint the natural coordinate system is polar centred at the joint, rather than Cartesian. It is straightforward, using a polar coordinate system, to demonstrate that all derivatives of the streamfunction are continuous functions of the azimuthal coordinate as the radial coordinate tends to zero and that the streamfunction is well behaved. Accordingly, for the general case we shall write $(2.16 a, b)$ in the form

$$
\begin{gathered}
L(\alpha, \bar{\omega}) F_{r}=R_{r}, \\
L(\alpha, \bar{\omega}) F_{c}=-R_{r}-(1-H(y-0+))(\bar{u} \underbrace{\Delta_{1}}_{O\left(\epsilon^{-2}\right)}-\frac{1}{R e}(\underbrace{\mathrm{i} \alpha \Delta_{1}}_{O\left(\epsilon^{-2}\right)}+\underbrace{\left.\Delta_{2}\right)}_{O\left(\epsilon^{-3}\right)}),
\end{gathered}
$$

where $R_{r}$ denotes the right-hand side of $(2.16 a), \Delta_{1}=f_{c, x x}(0,0)$ and $\Delta_{2}=f_{c, x x x}(0,0)$; $\Delta_{1}=0$ for clamped joints but is non-zero for pinned ones, whereas for $\Delta_{2}$ it is the other way round. This is somewhat similar to flow past a corner. It will be seen later on that the right-hand sides of $(2.18 a, b)$ are used only as weighted definite integrals in the domain $y=0$ to $y=1$; therefore, towards this end owing to the presence of the term $(1-H(y-0+))$, the last term on the right-hand side of $(2.18 b)$ makes little contribution. Hence the term involving $(1-H(y-0+))$ is neglected from hereon and not brought up in future discussions.

It is also necessary to determine the boundary conditions for the transformed streamfunction. For the centreline the end conditions at $x^{\prime}=0$ make no contribution and the boundary conditions $(2.3 a, b)$ become

$$
F_{r, c}^{\prime}(1 ; \alpha)=F_{r, c}^{\prime \prime \prime}(1 ; \alpha)=0 .
$$

Likewise for $x^{\prime}<0$, the wall boundary conditions $(2.4 a, b)$ become

$$
F_{r}(0 ; \alpha)=F_{r}^{\prime}(0 ; \alpha)=0 .
$$

For $x^{\prime}>0,(2.6)$ becomes

$$
F_{c}^{\prime}(0 ; \alpha)+\mathrm{i} \frac{\alpha \bar{u}^{\prime}(0)}{\bar{\omega}} F_{c}(0 ; \alpha)=0 .
$$


Taking the HFLT of the expression (2.11) for wall pressure, after minor rearrangement we obtain

$$
P_{c}(0 ; \alpha)=-\frac{\mathrm{i}}{\alpha \operatorname{Re}}\left[F_{c}^{\prime \prime \prime}(0 ; \alpha)-\alpha^{2} F_{c}^{\prime}(0 ; \alpha)\right],
$$

where $P_{c}$ is the HFLT of $\hat{p}_{w}$.

After substituting (2.22), the HFLT of (2.7) for the wall motion becomes

$$
\mathrm{i} \frac{\alpha^{2}}{\bar{\omega}}\left(\alpha^{4} B+K-\mathrm{i} \mathrm{d} \bar{\omega}-m \bar{\omega}^{2}\right) F_{c}(0 ; \alpha)+\frac{1}{R e}\left[F_{c}^{\prime \prime \prime}(0 ; \alpha)-\alpha^{2} F_{c}^{\prime}(0 ; \alpha)\right]=0 .
$$

A fully equivalent alternative formulation of (2.23) could be derived using the concept of admittance introduced by Landahl (1962) (see Carpenter \& Garrad 1985; Sen \& Arora 1988). This approach was used in the earlier versions of our theory (see Carpenter et al. 2002; Hegde 2002; Sen, Hegde \& Carpenter 2002, 2003; Carpenter $\&$ Sen 2003). The present approach has been adopted to show more clearly how the boundary conditions are treated in the analysis.

To proceed further, we take an important step forward to replace the near-field derived term $R_{r}$ on the right-hand side of $(2.18 a, b)$ by a discrete structure in $x$ located at $x^{\prime}=0$ having an amplitude distribution in $y$ as $\mathscr{F}(y)$. This step is analogous to replacing the thin viscous shock wave in compressible flow by an inviscid discontinuity. The function $\mathscr{F}(y)$ is called the wave driver function, or driver function. Thus we can restate the governing system of linear ordinary differential equations $(2.16 a, b)$ with boundary conditions $(2.19 a, b),(2.20 a, b),(2.21)$ and $(2.23)$ in the following more succinct forms:

$$
\begin{aligned}
& L(\alpha, \bar{\omega}) F_{r}=C \mathscr{F}(y), \\
& L(\alpha, \bar{\omega}) F_{c}=-C \mathscr{F}(y),
\end{aligned}
$$

where $\mathscr{F}(y)$ is an $O(1)$ function, the form of which remains to be determined, and $C$ is a constant, called later the driver strength, the order of magnitude and values of which remain to be determined. It will be shown later that $C \sim O\left(R e^{2 / 3}\right)$. Also note the opposite signs for $\mathscr{F}(y)$ respectively on the right-hand sides of $(2.24 a, b)$. This is because the wave driver is not a discrete 'source' in the conventional sense of the term. Rather, it is a novel discrete structure which is a 'half source cum half sink'; a 'source' for the outgoing wave and a 'sink' for the incoming wave. Other properties of the wave driver will be brought out later on. At the moment we note that the wave driver represents a strongly vortical structure, since it is a discretization of the (highest order) term $\bar{u}\left(\partial^{2} f\right) /\left.\left(\partial x^{2}\right)\right|_{x^{\prime}=0}$, in $(2.16 a, b)$.

The boundary conditions, for the rigid side and for the compliant side, may be gleaned from (2.19) to (2.23), which are respectively given as follows:

$$
\begin{gathered}
F_{r}^{\prime}(1)=F_{r}(1)^{\prime \prime \prime}=0, \quad F_{r}(0), F_{r}^{\prime}(0)=0 . \\
F_{c}^{\prime}(1)=F_{c}(1)^{\prime \prime \prime}=0 ; \\
F_{c}^{\prime}(0)+a(\alpha, \bar{\omega}) F_{c}(0)=0, \quad F_{c}^{\prime \prime \prime}(0)+b(\alpha, \bar{\omega}) F_{c}(0)=0 .
\end{gathered}
$$

The expressions $a(\alpha, \bar{\omega})$ and $b(\alpha, \bar{\omega})$ may easily be obtained from (2.21) and (2.23) respectively. The specific choices of $\alpha$ and $\omega$, in $a(\alpha, \omega)$ and $b(\alpha, \omega)$, will depend on specific situations. Note that $\bar{\omega}$ can be generalized to $\omega$. 
The key questions now are: How can a suitable driver function $\mathscr{F}$ be determined? And, given $\mathscr{F}$ how can the constant $C$ and the amplitude of the downstream far-field wave be determined? Before these questions are addressed in $\S 4$, we first apply our basic method immediately below in $\S 3$ to the much simpler case of finding the far-field solution for the case of a known driver function.

\section{Tollmien-Schlichting waves generated by a known driver}

In order to provide a relatively simple illustration of our basic approach we shall first consider the generation of TS waves in a rigid-wall plane Poiseuille flow. Let us suppose that the driver is located at $x=0$ and that it varies harmonically in time with frequency $\bar{\omega}$ just as in the case of the problem described in $\S 2$. In the far field downstream of the driver, providing $\bar{\omega}$ and the Reynolds number $R e$ lie within the region of instability, we would expect to find a spatially propagating TS wave. We shall show below that the amplitude of this downstream-propagating wave can be determined in terms of the known driver function without determining all the details of the near field. In many respects this problem is similar to the one involving the generation of a TS wave in a boundary layer by an idealized driver representing a vibrating ribbon. This problem was first investigated by Gaster (1965) and revisited by Ashpis \& Reshotko (1990). Here the problem is simpler because the flow is truly parallel and the continuous spectrum is absent.

The governing equation in the Fourier-Laplace domain is similar to (2.24), namely

$$
L(\alpha, \bar{\omega}) F=-C \mathscr{F}(y),
$$

which can be written in the form

$$
\left[L_{1}(\alpha)+\bar{\omega} L_{2}(\alpha)\right] F=-C \mathscr{F}(y),
$$

where

$$
L_{2}(\alpha) F=-\mathrm{i}\left(F^{\prime \prime}-\alpha^{2} F\right), \quad L_{1}(\alpha) F=\left[L(\alpha, \bar{\omega})-\bar{\omega} L_{2}(\alpha)\right] F
$$

where, in $(3.1 a, b)$, the minus sign appears on the right-hand side because we are interested in the domain $x>0$ and propagation downstream. Let us suppose that $C \mathscr{F}(y)$ represents a known function that is specified in advance. The far-field solution for $F$ is expected to take the form

$$
\frac{a^{\prime} \bar{\phi}(\bar{\alpha}, \bar{\omega})}{\alpha-\bar{\alpha}},
$$

where $\bar{\alpha}$ is the appropriate spatial rigid-wall eigenvalue and $\bar{\phi}$ the corresponding normalized eigenfunction. The object of the exercise is to determine a value of the amplitude $a$ of the far-field TS wave in the physical domain in terms of $C \mathscr{F}(y)$. Here $a$ is proportional to $a^{\prime}$. Further, since we will be confining ourselves to the rigid wall in this section, we will omit using the subscript ' $r$ '.

\subsection{The theory}

We need now to define formally the task at hand. It is actually to solve $(3.1 a)$ in a range of $\alpha$, with appropriate boundary conditions, and in the neighbourhood of $\bar{\alpha}$, so that in a natural way we are able to pick out the singular solution as $\alpha \rightarrow \bar{\alpha}$. Further, the words 'actually solve' are emphasized (rather than semi-quantitatively look at the dispersion relation only), because we would then be able to relate the amplitude of the far-field wave to that of the driver $C \mathscr{F}(y)$ itself. A very convenient 
way would be if we could expand $C \mathscr{F}(y)$ as a suitable eigenfunction expansion. In fact we were able to do just this, and also so, in a very convenient way. At this stage we introduce the concept of generalized temporal eigenstates. It will become clear why this is advantageous. For every complex value of $\alpha$ there exists an infinite number of eigenstates characterized by complex values of $\omega$. Let us denote these by

$$
\tilde{\phi}_{k}\left(\alpha, \tilde{\omega}_{k}\right), \quad k=1,2, \ldots, \infty ; \quad L\left(\alpha, \tilde{\omega}_{k}\right) \tilde{\phi}_{k}=0,
$$

such that $\tilde{\phi}_{k} \rightarrow \bar{\phi}_{k}$ and $\tilde{\omega}_{k} \rightarrow \bar{\omega}_{k}(k=1,2, \ldots, \infty)$ as $\alpha \rightarrow \bar{\alpha}$. Note that $\bar{\omega}_{1}(=\bar{\omega})$, which is the driver frequency, is actually real and is not the conventional temporal eigenvalue of hydrodynamic stability theory, although the absolute values will be close. We remember further that all the $\tilde{\phi}_{k}, \bar{\phi}_{k}$ are normalized eigenfunctions. Henceforth we shall use the following convention regarding notation: $\bar{q}$ will denote quantity, $q$, corresponding to the imposed or driver frequency $\bar{\omega}$ and far-field wavenumber $\bar{\alpha}$, whereas $\tilde{q}$ will denote quantities corresponding to the generalized temporal eigenstates corresponding to $\alpha \neq \bar{\alpha}$.

It is known for plane Poiseuille flow that any arbitrary transverse distribution of the perturbation streamfunction can be expressed as an infinite sum of the discrete temporal eigenfunctions, $\phi_{k}(k=1,2,3, \ldots)$, or, equivalently, of the set $\phi_{k}^{\prime \prime}-\alpha^{2} \phi_{k}$. No equivalent theorem appears to have been established for the spatial eigenfunctions. It is widely assumed (see for example Schmid \& Henningson 2001) that provided the appropriate set is chosen depending on whether one is computing upstream or downstream response, the perturbation streamfunction in this case also can be represented as an infinite sum of spatial eigenfunctions. It has been mentioned in the previous section that our driver function is not completely arbitrary, and is strongly vortical in content. There will be some discussions in the next section as to how the actual form of the driver function may be educed. It can however be seen from $(2.16 a, b)$ that the largest contribution to the driver should come from the term

$$
\left.C \mathscr{F}(y) \sim \bar{u} \frac{\partial^{2} f}{\partial x^{2}}\right|_{x=0} .
$$

Accordingly, it seems perfectly reasonable to represent the driver function in the present case in terms of the temporal eigenfunctions $\tilde{\phi}_{k}$. But before we proceed further we must hasten to answer the question 'why do we use a temporal eigenfunction expansion, in terms of $\tilde{\omega}_{k}$, rather than a spatial eigenfunction expansion?' The answer is direct and straightforward. There is an algebraic nonlinearity in $\alpha$ in the basic Orr-Sommerfeld equation (2.17) which renders a simple decomposition analogous to (3.1b) unfeasible. The advantage of using a decomposition like $(3.1 b)$ will become obvious in the next few steps because the solutions can be virtually read off. Hence we expand the driver function in the following form, at any $\alpha$, not necessarily equal to $\bar{\alpha}$ :

$$
C \mathscr{F}(y)=\sum_{k=1}^{\infty} \tilde{b}_{k} \tilde{g}_{k}
$$

where

$$
\tilde{g}_{k}=-\mathrm{i}\left(\tilde{\phi}_{k}^{\prime \prime}-\alpha^{2} \tilde{\phi}_{k}\right)
$$

The functions $\tilde{g}_{k}$ are called vorticity functions and have the useful property that they are orthogonal to the adjoint generalized eigenfunctions $\tilde{\theta}_{k}\left(\alpha, \tilde{\omega}_{k}\right)$ corresponding to 
$\tilde{\phi}_{k}\left(\alpha, \tilde{\omega}_{k}\right)$, so that

$$
\int_{0}^{1} \tilde{\theta}_{k} \tilde{g}_{j} \mathrm{~d} y=\delta_{j k} \int_{0}^{1} \tilde{\theta}_{k} \tilde{g}_{k} \mathrm{~d} y,
$$

where $\delta_{j k}=1$ when $j=k$ and is zero otherwise. Thus the coefficients in (3.3) are given by

$$
\tilde{b}_{k}=\frac{\int_{0}^{1} C \mathscr{F}(y) \tilde{\theta}_{k} \mathrm{~d} y}{\int_{0}^{1} \tilde{\theta}_{k} \tilde{g}_{k} \mathrm{~d} y} .
$$

Next we will develop the solution as $\alpha \rightarrow \bar{\alpha}$ and $\tilde{\omega}_{k} \rightarrow \bar{\omega}_{k}$. Particularly we will call $\bar{\omega}_{1}=\bar{\omega}$, where $\bar{\omega}$ is the actual physical driver frequency. Further we note from (2.17) that

$$
L_{\omega}\left(\alpha, \tilde{\omega}_{k}\right) \tilde{\phi}_{k}=L_{2}(\alpha) \tilde{\phi}_{k}=\tilde{g}_{k}, \quad L_{2}(\bar{\alpha}) \bar{\phi}_{k}=\bar{g}_{k} .
$$

We proceed further as follows:

$$
L(\alpha, \bar{\omega}) F=\left[L_{1}(\alpha)+\bar{\omega} L_{2}(\alpha)\right] F=-\sum_{k=0}^{\infty} \tilde{b}_{k} \tilde{g}_{k} .
$$

The above step is a precursor to the introduction of the generalized eigenfunctions $\tilde{\phi}_{k}(k=1,2,3, \ldots)$. We now expand $F$ in terms of $\tilde{\phi}_{k}$ (see $\left.(3.2 a, b)\right)$ as follows:

$$
F=\sum_{k=1}^{\infty} \tilde{a}_{k} \tilde{\phi}_{k}\left(\alpha, \tilde{\omega}_{k}\right)=\sum_{k=1}^{\infty} \frac{\tilde{a}_{k}^{\prime}}{\tilde{\omega}_{k}-\bar{\omega}_{1}} \tilde{\phi}_{k}\left(\alpha, \tilde{\omega}_{k}\right)
$$

where all $a_{k}^{\prime}$ are $O(1)$. Note that as $\alpha \rightarrow \bar{\alpha}$ then $\tilde{\omega}_{1} \rightarrow \bar{\omega}_{1}=\bar{\omega}$ and only the first term in the series is singular (as will be shown below, it has to take this form in order to recover the correct form of the far-field downstream wave after taking the inverse Fourier-Laplace transform); it is only for convenience that the coefficients of the remaining terms are written with denominators in the forms of differences in complex frequency. We now substitute for $F$ from (3.10) into (3.9). Thereafter, for each value of $k$ we have

$$
\tilde{a}_{k} L(\alpha, \bar{\omega}) \tilde{\phi}_{k}=\tilde{a}_{k} \underbrace{\left[L_{1}(\alpha)+\tilde{\omega}_{k} L_{2}(\alpha)\right] \tilde{\phi}_{k}}-\left(\tilde{\omega}_{k}-\bar{\omega}\right) \tilde{a}_{k} \tilde{g}_{k}=\tilde{b}_{k} \tilde{g}_{k},
$$

where, by definition, the terms in underbrace, viz.

$$
\left[L_{1}(\alpha)+\tilde{\omega}_{k} L_{2}(\alpha)\right] \tilde{\phi}_{k}=L\left(\alpha, \tilde{\omega}_{k}\right) \tilde{\phi}_{k}=0 .
$$

We now get a formal solution for $\tilde{a}_{k}$, from $(3.11 a, b)$, as follows:

$$
\tilde{a}_{k}=\frac{\tilde{b}_{k}}{\left(\tilde{\omega}_{k}-\bar{\omega}\right)}
$$

and remembering (3.10) we have

$$
\tilde{a}_{k}^{\prime}=\tilde{b}_{k} .
$$

It therefore appears that the solution to (3.9) can virtually be read off, in terms of the generalized eigenstates of $L\left(\alpha, \tilde{\omega}_{k}\right) \tilde{\phi}_{k}=0$. Now we go to an important limit when $\alpha \rightarrow \bar{\alpha}$ and $\tilde{\omega}_{1} \rightarrow \bar{\omega}_{1}(=\bar{\omega})$. This leads to the singular term in (3.1) for $k=1$. Moreover, as $\alpha \rightarrow \bar{\alpha}, \tilde{\phi}_{1} \rightarrow \bar{\phi}$ and $\tilde{\theta}_{1} \rightarrow \bar{\theta}$. Also, the coefficients $\tilde{a}_{k}^{\prime}(k>1)$ are non-singular and therefore have no contribution in the inversion of the pole, and thus do not need to be actually evaluated for determining the far-field solution. 
The singular solution $F_{s}$, corresponding to the far-field downstream TS wave, is given by setting $k=1$ in (3.10), and going to the limit $\alpha \rightarrow \bar{\alpha}$, which also gives $\tilde{\phi}_{1} \rightarrow \bar{\phi}$. Hence we obtain the following:

$$
F_{s}=\bar{\phi} \frac{I_{2}}{\left(\tilde{\omega}_{1}-\bar{\omega}\right) I_{1}}=\bar{\phi} \frac{I_{2}}{(\alpha-\bar{\alpha}) c_{g} I_{1}},
$$

where

$$
I_{1}=\int_{0}^{1} \bar{\theta} \bar{g} \mathrm{~d} y, \quad I_{2}=\int_{0}^{1} C \mathscr{F} \bar{\theta} \mathrm{d} y, \quad c_{g}=\frac{\mathrm{d} \omega}{\mathrm{d} \alpha},
$$

where $c_{g}$ is the complex group velocity.

In order to determine the far-field solution in the physical plane we need to carry out an inverse Fourier-Laplace transformation of $(3.13 a)$. Calling the Fourier-Laplace inverse $F^{-}$, we have

$$
F^{-}\left[F_{s}\right]=f(x, y), \quad f(x, y) \mathrm{e}^{-\mathrm{i} \bar{\omega} t}=a \bar{\phi} \mathrm{e}^{\mathrm{i}(\bar{\alpha} x-\bar{\omega} t)},
$$

where $a$ is the amplitude of the normalized far-field eigenfunction $\bar{\phi}$. Also, from (3.13-3.14) we have the inverse transform as

$$
\begin{gathered}
a \bar{\phi} \mathrm{e}^{\mathrm{i}(\bar{\alpha} x-\bar{\omega} t)}=\frac{\bar{\phi} \mathrm{e}^{-\mathrm{i} \bar{\omega} t}}{2 \pi c_{g}} \frac{I_{2}}{I_{1}} \int_{C_{r}} \frac{\mathrm{e}^{\mathrm{i} \alpha x}}{\alpha-\bar{\alpha}} \mathrm{d} \alpha, \\
\int_{C_{r}} \frac{\mathrm{e}^{\mathrm{i} \alpha x}}{\alpha-\bar{\alpha}} \mathrm{d} \alpha=2 \mathrm{i} \pi \mathrm{e}^{\mathrm{i} \bar{\alpha} x},
\end{gathered}
$$

where $C_{r}$ is an appropriate contour of integration. Therefore, we have

$$
a \bar{\phi} \mathrm{e}^{\mathrm{i}(\bar{\alpha} x-\bar{\omega} t)}=\frac{\mathrm{i}}{c_{g}} \frac{I_{2}}{I_{1}} \bar{\phi} \mathrm{e}^{\mathrm{i}(\bar{\alpha} x-\bar{\omega} t)} .
$$

Hence the all important amplitude $a$ of the normalized far-field eigenfunction $\bar{\phi}$ is given as

$$
a=\frac{\mathrm{i} I}{c_{g}}, \quad I=\frac{I_{2}}{I_{1}} .
$$

Thus the amplitude of the downstream TS wave is now given in terms of the driver function by $(3.1 a, b, c, d)$. In the end we see that the procedure comes out to be astonishingly simple. Given a driver function $\mathscr{F}(y)$ (of strength $C$ ) all we need to determine the amplitude $a$ of the far-field solution are the integrals $I_{1}$ and $I_{2}$ and the group velocity $c_{g}$.

\subsection{Application of theory to a known driver}

To illustrate the application of the theoretical approach outlined above let us consider a driver that takes the form of an oscillating velocity imposed at the wall taking the form

$$
v_{w}=\hat{v}_{w} \delta(x) \mathrm{e}^{\mathrm{i} \omega t} .
$$

A driver of this form was used by Gaster (1965). For numerical simulations it is necessary to approximate the delta function and write the driver as

$$
\frac{\hat{v}_{w}}{\ell \sqrt{\pi}} \mathrm{e}^{-(x / \ell)^{2}} \text {. }
$$


Using the methods described in Davies \& Carpenter (1997) we carried out a numerical simulation using this driver with $R e=12000, \bar{\omega}=0.24, \ell=0.125$ and $\hat{v}_{w}=\ell \sqrt{\pi}$. As expected, a growing TS wave was generated downstream with a complex wavenumber $\alpha=(1.0317,-0.0093)$ in good agreement with the values we determined from hydrodynamic stability theory. This far-field solution,

$$
v(x, y, t)=-\frac{\partial \psi}{\partial x}=\underbrace{-\mathrm{i} a \alpha \bar{\phi}_{r}(y)}_{\hat{v}} \mathrm{e}^{\mathrm{i}(\alpha x-\omega t)},
$$

can be extrapolated backwards to the origin in order to determine the corresponding amplitude of the normal velocity on the centreline, namely $-\mathrm{i} a \alpha$, provided we adopt a normalization whereby $\bar{\phi}_{r}(1)=(1.0,0.0)$. (Again we introduce the generic subscript ' $r$ ' for the rigid wall). Hence we obtain the value of the amplitude of the streamfunction as $a=(-0.19,-0.06)=0.20 \mathrm{e}^{\mathrm{i} 1.1 \pi}$.

We shall now calculate this quantity using our theoretical result, $(3.15 d, e)$, with the driver function approximated in the form given in (3.3). It will be shown in $\S 5$ that this approximate form relies for its validity on the width of the driver region being sufficiently narrow to drop all the terms but one on the right-hand side of (2.16). Note, however, that the width of the present driver, namely $\ell=0.125$, is an order of magnitude greater than that of the virtual wave driver in $\S 5$. Thus, although the neglected terms on the right-hand side of (2.16) will remain secondary, their contribution cannot be expected to be completely negligible.

A further subtlety concerns the approximation of the delta function. If we were actually using a delta function then $\mathrm{d} \hat{v}_{w} / \mathrm{d} x=\delta^{\prime}(x)$. Thus we need to find a suitable approximation to $\delta^{\prime}(x)$ that corresponds to the numerical simulation. This can be given from the form in (3.16), from which it follows that the driver wall-normal perturbation velocity is given by

$$
v(x, y)=\frac{\hat{v}(y)}{\ell \sqrt{\pi}} \mathrm{e}^{-(x / \ell)^{2}} \quad(\simeq \hat{v}(y) \delta(x)) .
$$

It may be seen in (2.16) earlier, and also later on in discussions following (5.4), that when a stationary narrow vortical structure exists in the fluid side, the largest order term is as given by (3.3). The vibrating ribbon-like disturbance, given by (3.18), is also expected to create a vortical structure above itself in the fluid side. The existence of such a vortical structure is confirmed by the numerical simulations. Remembering in (3.3) that the quantity $f$ is the streamfunction, we therefore have the driver function approximated as follows:

$$
\bar{u} \frac{\partial^{2} f}{\partial x^{2}} \simeq \bar{u}(y) \hat{v}(y) \underbrace{\frac{1}{\ell \sqrt{\pi}} \frac{\partial}{\partial x}\left(\mathrm{e}^{-(x / \ell)^{2}}\right)}_{\simeq \delta^{\prime}(x)} .
$$

Thus the generalized function $\delta^{\prime}(x)$ is approximated and replaced by the regular function

$$
\frac{1}{\ell \sqrt{\pi}} \frac{\partial}{\partial x}\left(\mathrm{e}^{-(x / \ell)^{2}}\right)=-\frac{2}{\sqrt{\pi} \ell^{2}}\left(\frac{x}{\ell}\right) \mathrm{e}^{-(x / \ell)^{2}} .
$$

The maximum value of this function occurs at $x / \ell=1 / \sqrt{2}$, and we take this maximum value as our approximation to $\delta^{\prime}(x)$. We also evaluate $\partial \hat{v} / \partial x$ at this point rather than at the origin where $\partial \hat{v}_{w} / \partial x=0$. 
The variation in $y$ of $\partial \hat{v} / \partial x$ and $\bar{u} \partial \hat{v} / \partial x$ are obtained from the numerical simulations, and are plotted in figure 5; the corresponding eigenfunction $\bar{\phi}_{r}$, adjoint eigenfunction $\bar{\theta}_{r}$ and the vorticity function $\bar{g}_{r}$ are plotted in figures 6 and 7 respectively. The value of the integral in the numerator of (3.7), i.e $(3.13 c)$, is determined numerically using the data given in figure 5 and a complex value of $(0.308,0.153)$ obtained, when the approximation (3.18), for $\delta^{\prime}(x)$, is kept in. The integral in the denominator of (3.7), i.e (3.13d), is similarly found to have a complex value of $(-1.847,6.285)$, and the complex group velocity is obtained as $c_{g}=(0.325,-0.042)$. Thus $(3.15 a)$ gives $a \simeq(-0.17,-0.045) \simeq 0.18 \mathrm{e}^{\mathrm{i} 1.07 \pi}$. Given that the driver is relatively wide, the theoretical value of $a$ seems to be in reasonable agreement with the value of $a=0.20 \mathrm{e}^{\mathrm{i} 1.1 \pi}$ given above, which was determined from the numerical simulation.

\section{The wave driver theory}

\subsection{Characteristics of the wave driver}

We begin this section by listing out some of the important characteristics of the wave driver. Some of these have already come in the previous discussions. Some more characteristics will come forth in the subsequent discussions. The main characteristics are the following:

1. The wave driver is an idealized discrete structure, formed in the fluid side, at the junction of two different wave-bearing media, like rigid-compliant or compliant-rigid walls.

2. The wave driver is an idealized discrete structure that purports to capture some important features of the narrow viscous interface that is known to form when a disturbance wave crosses a junction. Thus the wave driver is associated with an incoming wave and an outgoing wave, respectively belonging to the different wavebearing media on either side of the junction, like rigid-compliant or compliant-rigid.

3. The wave driver is strongly vortical in content and is neither associated with nor causes any wall displacement at its point of location at the junction.

4. The wave driver is a discrete structure hitherto not used in any past work. It is actually a 'half source cum half sink'. It behaves like a sink for the incoming wave, and like a source for the outgoing wave. The 'source' part of the wave driver is similar to the classically known, and used, sources, for example the vibrating ribbon, from which near-neutral waves (both amplified or damped) can tune out and reach the far field. The 'sink' part is something new and its precise nature will come out in the course of subsequent discussions.

5. The wave driver cannot support, nor is by itself responsible for, any wave reflection. This is because it is essentially a structure in the fluid side, and there is no natural barrier in the fluid side that can support a wave reflection.

6. Both the incoming wave and the outgoing wave at a wave driver must be strongly vortical; it is then that the vortical structure, viz. the wave driver, actually comes to exist.

7. The wave driver, being strongly vortical in content, cannot be formed by an incoming near-inviscid wave, i.e. by an incoming wave that has very small vorticity. Likewise, a near-inviscid, or nearly non-vortical, wave cannot tune out of an already existing wave driver. Inviscid waves come to exist because of wall displacement or can tune out from a wall-displacement source (cf. Lucey et al. 2003).

8. When a wave driver has already come to exist, due to an incoming and outgoing vortical wave pair, there is at least a theoretical possibility of other outgoing far-field 
waves tuning out of this existing wave driver. However, such specific possible cases need to be considered individually and carefully.

9. Across a wave driver at a junction the direction of energy propagation must be consistent. This point will be discussed in greater detail later on in this section.

10. Conservation of energy between the incoming and outgoing waves across the wave driver is not addressed to in the present wave driver theory. It is well known from the simulations in Davies \& Carpenter (1997) that the near field at a junction comprises complex production and dissipation processes. However, detailed study of the near field is not part of the present work.

\subsection{The incoming rigid-side TS wave at the leading-edge junction}

Next we consider the incoming rigid-side TS wave that approaches the leading-edge junction. The analysis is very similar to what has been outlined earlier in $\S 3.1$, except that there is a sign reversal throughout because the relevant base equation is $(2.24 a)$. Using the generic subscript ' $r$ ' for the rigid side, we have the amplitude $a_{r}$ of the rigid side, in relation to the driver function $C \mathscr{F}(y)$, given from $(3.15 d, e)$ as follows:

$$
\begin{gathered}
a_{r}=-\frac{\mathrm{i} C I_{r}}{c_{g r}}, \quad I_{r}=\frac{I_{2 r}}{I_{1 r}}, \\
I_{1 r}=\int_{0}^{1} \bar{\theta}_{r} \bar{g}_{r} \mathrm{~d} y, \quad I_{2 r}=\int_{0}^{1} \mathscr{F} \bar{\theta}_{r} \mathrm{~d} y, \quad c_{g r}=\frac{\mathrm{d} \omega}{\mathrm{d} \alpha},
\end{gathered}
$$

where $c_{g r}$ is the group velocity of the rigid-side TS wave. Note the slight difference in forms between (3.13c) and (4.1d) and between (3.15d) and (4.1a), $C$ having been kept outside the integrals in $(4.1 a)$ and $(4.1 d)$. If we specify the rigid-side wave amplitude $a_{r}$ to be the reference amplitude, we may put $a_{r}=1$. Then, we are able to estimate the driver strength $C$, from $(4.1 a-e)$, as follows:

$$
C=\frac{\mathrm{i} c_{g r}}{I_{r}} .
$$

A rough estimate of the order of $C$ may be made using the Tollmien scale for the viscous critical layer which gives the width of the viscous critical layer in the OrrSommerfeld solution, $\epsilon_{c}$, as $\epsilon_{c} \sim O\left(R e^{-1 / 3}\right)$. This gives the order of $\bar{\phi}_{\underline{r}}^{\prime \prime}$ as $\bar{\phi}_{r}^{\prime \prime} \sim O\left(R e^{2 / 3}\right)$. Further, $\bar{g}_{r}$ is dominated by $\bar{\phi}_{r}^{\prime \prime}$, and also it is well known that $\bar{\theta}_{r} \sim \bar{\phi}_{r}^{\prime \prime}$. Hence it is easy to estimate from (4.1) and (4.2) that the order of the driver strength $C$ is given as

$$
C \sim O\left(R^{2 / 3}\right)
$$

The adjoint Orr-Sommerfeld equation for the adjoint eigenfunction $\theta$ together with its boundary conditions over a compliant wall are given in the Appendix.

\subsection{Wave driver theory for the compliant side}

We now focus attention on the compliant side. The basic equation in the FourierLaplace domain for the compliant side is given from $(2.24 a, b)$ as follows:

$$
L(\alpha, \bar{\omega}) F_{c}=\mp C \mathscr{F}(y),
$$

where in $\mp$, the '-' sign signifies that the wave is evolving from the leading-edge junction, and the ' + ' sign signifies that the wave is coming into the trailing-edge junction. The task at hand is similar to that in $\S 3.1$. We need to be able to solve $(4.4 a, b)$ for values of $\alpha$ in the neighbourhood of $\bar{\alpha}$, so that the singular and regular solutions of $F_{c}$, respectively $\left[F_{c}\right]_{s}$ and $\left[F_{c}\right]_{r}$, come out in a natural way as $\alpha \rightarrow \bar{\alpha}$, with $F_{c}=\left[F_{c}\right]_{s}+\left[F_{c}\right]_{r}$. The added complication that arises for the compliant case is because 
of the compliant-wall boundary conditions, which need to be considered very carefully. We must also note that $F_{c}$ can have only one unique set of boundary conditions. Thus, if we think of an eigenfunction expansion for $F_{c}$, like $\tilde{\phi}_{c k}(k=1,2,3, \ldots)$, then, all the $\tilde{\phi}_{c k}$ need to satisfy the same set of boundary conditions. For the rigid-wall case this was not a problem because all the $\tilde{\phi}_{r k}$ satisfy the same homogeneous boundary conditions at the wall, viz. $\tilde{\phi}_{r k}(0), \tilde{\phi}_{r k}^{\prime}(0)=0$. For the compliant case the boundary conditions are given by $(2.26 a-d)$, reproduced here below:

$$
\begin{gathered}
F_{c}^{\prime}(1)=F_{c}(1)^{\prime \prime \prime}=0, \\
F_{c}^{\prime}(0)+a(\alpha, \omega) F_{c}(0)=0, \quad F_{c}^{\prime \prime \prime}(0)+b(\alpha, \omega) F_{c}(0)=0 .
\end{gathered}
$$

It was mentioned in the discussions following $(2.26 c, d)$ that 'the specific choices of $\alpha$ and $\omega$, in $a(\alpha, \omega)$ and $b(\alpha, \omega)$, will depend on specific situations'. It is time now to address this issue. As $\alpha \rightarrow \bar{\alpha}$, then $\alpha$ and $\omega$, in $a(\alpha, \omega)$ and $b(\alpha, \omega)$, should also approach $\bar{\alpha}$ and $\bar{\omega}$. There are two routes by which this may happen, respectively called 'Route A' and 'Route B'. These are discussed next.

Route A: Suppose that $\left(\alpha, \tilde{\omega}_{c 1}\right)$ is a compliant (and physically possible) eigenvalue pair, with the corresponding eigenfunction $\tilde{\phi}_{c 1}$. This is an eigensolution neighbouring the far-field eigenvalue pair $(\bar{\alpha}, \bar{\omega})$. Also, suppose, as previously described in $\S 3$ earlier, we have a system of eigenfunctions $\tilde{\phi}_{c k}(k=1,2,3, \ldots)$ satisfying the following equation and boundary conditions:

$$
\begin{gathered}
L\left(\alpha, \tilde{\omega}_{c k}\right) \tilde{\phi}_{c k}=0, \quad k=1,2,3, \ldots, \\
\tilde{\phi}_{c k}^{\prime}(1)=\tilde{\phi}_{c k}(1)^{\prime \prime \prime}=0, \\
\tilde{\phi}_{c k}^{\prime}(0)+a\left(\alpha, \tilde{\omega}_{c 1}\right) \tilde{\phi}_{c k}(0)=0, \quad \tilde{\phi}_{c k}^{\prime \prime \prime}(0)+b\left(\alpha, \tilde{\omega}_{c 1}\right) \tilde{\phi}_{c k}(0)=0 .
\end{gathered}
$$

The key feature in the above wall boundary conditions $(4.7 c, d)$ is that all the wall boundary conditions, for all the $\tilde{\phi}_{c k}(k \geqslant 1)$, correspond to $a, b$ being frozen to $a\left(\alpha, \tilde{\omega}_{c 1}\right)$ and $b\left(\alpha, \tilde{\omega}_{c 1}\right)$. This way, only $\tilde{\phi}_{c 1}$ is a physically possible eigenfunction. The remaining eigenfunctions $\tilde{\phi}_{c k}(k>1)$ are non-physical eigenfunctions that have been introduced to be able to solve $(4.4 a, b)$, for $F_{c}$, in Fourier-Laplace space. We now write an eigenfunction expansion for $F_{c}$, on the lines of $(3.10 a, b)$, as follows:

$$
F=\sum_{k=1}^{\infty} \tilde{a}_{c k} \tilde{\phi}_{c k}\left(\alpha, \tilde{\omega}_{c k}\right)=\sum_{k=1}^{\infty} \frac{\tilde{a}_{c k}^{\prime}}{\tilde{\omega}_{c k}-\bar{\omega}_{c 1}} \tilde{\phi}_{c k}\left(\alpha, \tilde{\omega}_{c k}\right) .
$$

Note that the expansion retrieves the singular and regular parts of $F_{c}=\left[F_{c}\right]_{s}+\left[F_{c}\right]_{r}$, viz. $\left[F_{c}\right]_{s},\left[F_{c}\right]_{r}$ respectively, as $\alpha \rightarrow \bar{\alpha}_{c}$, when also $\tilde{\omega}_{c 1} \rightarrow \bar{\omega}, \tilde{\phi}_{c k} \rightarrow \bar{\phi}_{c k}$ and $\tilde{\phi}_{c 1} \rightarrow$ $\bar{\phi}_{c 1}\left(=\bar{\phi}_{c}\right)$. The other point to note is that, in view of $(4.7 c, d)$ and $(4.7 a, b)$, both $\left[F_{c}\right]_{s}$ and $\left[F_{c}\right]_{r}$ satisfy the same wall boundary conditions and same outer boundary conditions. The solution is therefore legitimate and approaches the limit $\alpha \rightarrow \bar{\alpha}_{c}$ in a natural way.

The rest of the analysis is similar to that in $\S 3.1$, and in $\S 4.1$ above, for the rigid case, except that the generic subscript ' $c$ ' is used throughout. Also the equivalents of (3.4) for the expansion of $\mathscr{F}(y)$ and the Fourier-Laplace inversion integral (3.15b) are given respectively as

$$
\begin{gathered}
\mathscr{F}(y)=\sum_{k=1}^{\infty} \tilde{b}_{c k} \tilde{g}_{c k}, \\
\int_{C_{c}} \frac{\mathrm{e}^{\mathrm{i} \alpha x}}{\alpha-\bar{\alpha}_{c}} \mathrm{~d} \alpha=2 \mathrm{i} \pi \mathrm{e}^{\mathrm{i} \bar{\alpha}_{c} x},
\end{gathered}
$$


where $C_{c}$ is an appropriate integration contour for the Fourier-Laplace inversion integral in (4.10). The final answer for the amplitude $a_{c}$ for the far-field compliant wave is given similarly as in $(4.1 a, b)$ and $(4.1 c-e)$ for the rigid case, that is

$$
\begin{gathered}
a_{c}=\frac{\mathrm{i} C I_{c}}{c_{g c}}, \quad I_{c}=\frac{I_{2 c}}{I_{1 c}}, \\
I_{1 c}=\int_{0}^{1} \bar{\theta}_{c} \bar{g}_{c} \mathrm{~d} y, \quad I_{2 c}=\int_{0}^{1} \mathscr{F} \bar{\theta}_{c} \mathrm{~d} y, \quad c_{g c}=\frac{\mathrm{d} \omega}{\mathrm{d} \alpha},
\end{gathered}
$$

where $c_{g c}$ is the regular group velocity for the far-field compliant wave. For the present 'Route A', where the pair $\left(\alpha, \tilde{\omega}_{c 1}\right)$ is a neighbouring physical eigenvalue to the far-field compliant eigenvalue pair $\left(\bar{\alpha}_{c}, \bar{\omega}\right)$, it is obvious that the regular group velocity $c_{g c}$ for the far-field compliant wave needs to be used in (4.11a). Also the sign reversal in $(4.11 a)$, as compared to $(4.1 a)$ for the rigid case, is because it is being assumed that we are at the leading-edge junction where the rigid-side wave is the incoming wave and the compliant-side wave is the outgoing wave.

We now have a method of determining the amplitude ratio $\lambda_{c}$ of the compliant-side to rigid-side amplitude. This is also called the 'jump in amplitude'. Remembering that we determined the driver strength $C$ in (4.2) above, by keeping the rigid-side amplitude $a_{r}$ as $a_{r}=1$, we have the amplitude ratio $\lambda_{c}$ given as follows:

$$
\lambda_{c}=\frac{a_{c}}{a_{r}}=\frac{\mathrm{i} C I_{c}}{c_{g c}}=-\frac{c_{g r}}{c_{g c}} \frac{I_{c}}{I_{r}} .
$$

In closing we mention that we have considered the above example with reference to the leading-edge junction. However, at this point we are not saying that Route A is appropriate, or not, to the leading-edge junction. This point will come up in a natural way after Route B is discussed.

Route $B$ : We begin the description of Route $\mathrm{B}$ by introducing the generic circumflex accent, i.e. '( $\left.{ }^{(}\right)$', for all the associated quantities in this route. Analogous to the case in Route $\mathrm{A}$ we have a system of eigenfunctions $\hat{\phi}_{c k}(k=1,2,3, \ldots)$ satisfying the following equation and boundary conditions:

$$
\begin{gathered}
L\left(\alpha, \hat{\omega}_{c k}\right) \hat{\phi}_{c k}=0, \quad k=1,2,3, \ldots, \\
\hat{\phi}_{c k}^{\prime}(1)=\hat{\phi}_{c k}(1)^{\prime \prime \prime}=0, \\
\hat{\phi}_{c k}^{\prime}(0)+a(\bar{\alpha}, \bar{\omega}) \hat{\phi}_{c k}(0)=0, \quad \hat{\phi}_{c k}^{\prime \prime \prime}(0)+b(\bar{\alpha}, \bar{\omega}) \hat{\phi}_{c k}(0)=0 .
\end{gathered}
$$

The key feature in the Route B wall boundary conditions $(4.14 c, d)$ is that all the wall boundary conditions, for all the $\hat{\phi}_{c k}(k \geqslant 1)$, correspond to $a, b$ being frozen to $a(\bar{\alpha}, \bar{\omega})$ and $b(\bar{\alpha}, \bar{\omega})$. This way, none of the eigenfunctions $\hat{\phi}_{c k}(k \geqslant 1)$ is a physically possible eigenfunction. Also we remember that $\hat{\phi}_{c 1} \rightarrow \bar{\phi}_{c}$, and $\hat{\omega}_{c 1} \rightarrow \bar{\omega}$ when $\alpha \rightarrow \bar{\alpha}_{c}$. Moreover, we emphasize that $\hat{\phi}_{c 1}$ is also not a physically possible eigenfunction when $\alpha \neq \bar{\alpha}_{c}$. Again, the fact that all the $\hat{\phi}_{c k}$ are not physical eigenfunctions is not of much consequence because these non-physical eigenfunctions have been introduced to be able to solve $(4.4 a, b)$, for $F_{c}$, in Fourier-Laplace space. The rest of the mathematics is very similar to Route A and is reproduced below for the sake of reference. As before, we write an eigenfunction expansion for $F_{c}$ as follows:

$$
F_{c}=\sum_{k=1}^{\infty} \hat{a}_{c k} \hat{\phi}_{c k}\left(\alpha, \hat{\omega}_{c k}\right)=\sum_{k=1}^{\infty} \frac{\hat{a}_{c k}^{\prime}}{\hat{\omega}_{c k}-\bar{\omega}_{c 1}} \hat{\phi}_{c k}\left(\alpha, \hat{\omega}_{c k}\right) .
$$


Again the above expansion retrieves the singular and regular parts of $F_{c}$, viz. $\left[F_{c}\right]_{s},\left[F_{c}\right]_{r}$ respectively, as $\alpha \rightarrow \bar{\alpha}_{c}$, when also $\hat{\omega}_{c 1} \rightarrow \bar{\omega}, \hat{\phi}_{c k} \rightarrow \bar{\phi}_{c k}$ and $\hat{\phi}_{c 1} \rightarrow$ $\bar{\phi}_{c 1}\left(=\bar{\phi}_{c}\right)$. Again one may note that, in view of $(4.14 c, d)$ and $(4.14 a, b)$, both $\left[F_{c}\right]_{s}$ and $\left[F_{c}\right]_{r}$ satisfy the same wall boundary conditions and same outer boundary conditions. The solution is therefore legitimate and approaches the limit $\alpha \rightarrow \bar{\alpha}_{c}$ in a natural way. Also (4.9) in Route A carries over in a natural way to Route B as follows:

$$
\mathscr{F}(y)=\sum_{k=1}^{\infty} \hat{b}_{c k} \hat{g}_{c k} .
$$

Also (4.10) for Route A is identical in Route B.

However, the major difference between Route A and Route B is in the group velocity. This point needs careful attention. If we look at the equivalent of $(3.13 a)$, as adapted to the present case, we have

$$
\begin{gathered}
{\left[F_{c}\right]_{s}=\bar{\phi}_{c} \frac{C \hat{I}_{c 2}}{\left(\hat{\omega}_{c 1}-\bar{\omega}\right) \hat{I}_{c 1}}} \\
\hat{I}_{c 1}=\int_{0}^{1} \bar{\theta}_{c} \bar{g}_{c} \mathrm{~d} y, \quad \hat{I}_{c 2}=\int_{0}^{1} \mathscr{F}_{\bar{\theta}} \mathrm{d} y .
\end{gathered}
$$

Now the question is how do we relate $\delta \omega=\left(\hat{\omega}_{c 1}-\bar{\omega}\right)$ to $\delta \alpha=\left(\alpha-\alpha_{c}\right)$ so that the singular solution $\left[F_{c}\right]_{s}$ may be inverted to the physical domain. Can we use the compliant-side group velocity $c_{g c}$ as we did in the case of Route A? The answer to the last question is a plain and simple 'no', because $\hat{\phi}_{c 1}$ is no doubt a neighbouring eigenfunction, but it is not a neighbouring physical eigenfunction.

We set to answer the above question, regarding group velocity, by considering two neighbouring eigenvalues and eigenfunctions. Dropping the generic subscript ' $c$ ' and the circumflex accent $\left(^{\wedge}\right)$ we write the pair of neighbouring eigenvalues as $(\bar{\alpha}, \bar{\omega})$ and $(\alpha, \omega)$, and the corresponding eigenfunctions as $\bar{\phi}$ and $\phi$. The above quantities are related as below:

$$
\alpha=\bar{\alpha}+\delta \alpha, \quad \omega=\bar{\omega}+\delta \omega, \quad \phi=\bar{\phi}+\delta \phi .
$$

Both $\bar{\phi}$ and $\phi$ are solutions of the compliant Orr-Sommerfeld equation, satisfying the boundary conditions $(4.14 a, b)$ and $(4.14 c, d)$. In view of $(4.19 c), \delta \phi$ also satisfies the same boundary conditions. We therefore write the equation for $\phi$ as a variation of that for $\bar{\phi}$ as follows:

$$
L(\bar{\alpha}, \bar{\omega}) \bar{\phi}=0, \quad L(\alpha, \omega) \phi=0, \quad L(\bar{\alpha}+\delta \alpha, \bar{\omega}+\delta \omega)(\bar{\phi}+\delta \phi)=0 .
$$

Remembering (4.20a), we may now expand (4.20c) as follows:

$$
L(\bar{\alpha}, \bar{\omega}) \delta \phi+\delta \alpha L_{\alpha}(\bar{\alpha}, \bar{\omega}) \bar{\phi}+\delta \omega L_{\omega}(\bar{\alpha}, \bar{\omega}) \bar{\phi}=0 .
$$

We now look at the solvability of (4.21). Had the boundary conditions for $\bar{\phi}, \phi$ and $\delta \phi$ been different, as was the case in Route A, then (4.21) would not be of much use to us, with different terms in the differential equation satisfying different boundary conditions. However, for the present case (Route B), all of $\bar{\phi}, \phi$ and $\delta \phi$ satisfy the same set of boundary conditions. (Incidentally this is so for the rigid-wall case also). Therefore we may obtain the solvability condition for (4.21) as follows:

$$
\delta \alpha \int_{0}^{1} \bar{\theta} L_{\alpha}(\bar{\alpha}, \bar{\omega}) \bar{\phi} \mathrm{d} y+\delta \omega \int_{0}^{1} \bar{\theta} L_{\omega}(\bar{\alpha}, \bar{\omega}) \bar{\phi} \mathrm{d} y=0 .
$$


(a)

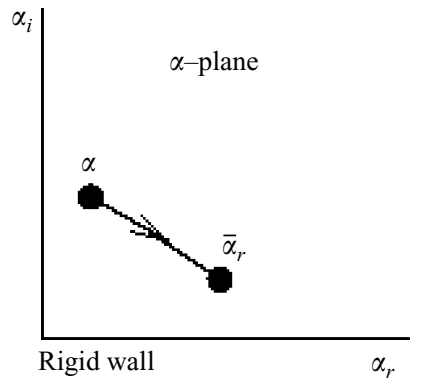

(b)

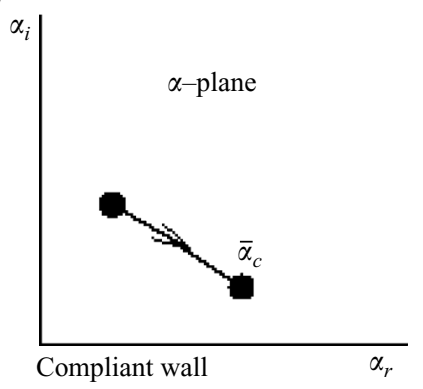

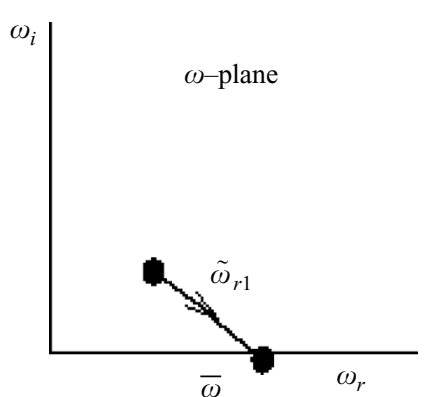

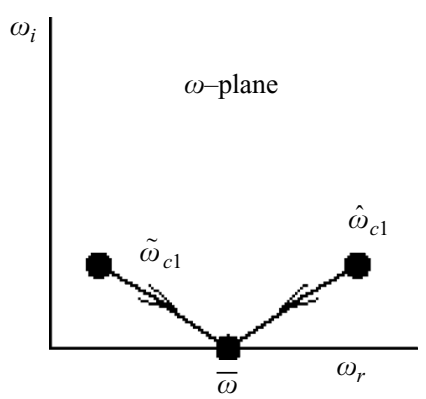

FIGURE 4. A schematic sketch illustrating the relationship between the eigenstates in the $\alpha$-plane and the generalized temporal eigenstates in the $\omega$-plane. Subscripts ' $r$ ' and ' $i$ ' refer to real and imaginary parts.

The relationship between $\delta \alpha$ and $\delta \omega$, obtained from (4.22), gives us the pseudo group velocity $c_{p}$, the expression for which is given below:

$$
c_{p}=\frac{\delta \omega}{\delta \alpha}=-\frac{\int_{0}^{1} \bar{\theta} L_{\alpha}(\bar{\alpha}, \bar{\omega}) \bar{\phi} \mathrm{d} y}{\int_{0}^{1} \bar{\theta} L_{\omega}(\bar{\alpha}, \bar{\omega}) \bar{\phi} \mathrm{d} y} .
$$

Finally, the equation for the jump $\lambda_{c}$ for the present case, i.e. Route $B$, is given similarly as in $(4.12 c)$ as follows:

$$
\lambda_{c}=-\frac{c_{g r}}{c_{p}} \frac{I_{c}}{I_{r}}
$$

where $c_{p}$ has been put in place of $c_{g c}$.

Incidentally, for the rigid-wall case, $c_{p}$ and $c_{g}$ are the same; but for the compliantwall case these are different. The pseudo group velocity is a new concept, the physical ramifications of which will be described in the next two subsections.

The two routes by which the singular solution is approached for the compliant-wall cases as $\alpha \rightarrow \bar{\alpha}_{c}$, viz. $\tilde{\omega}_{c 1} \rightarrow \bar{\omega}$ for Route $\mathrm{A}$ and $\hat{\omega}_{c 1} \rightarrow \bar{\omega}$ for Route $\mathrm{B}$, are shown in figure $4(b)$. Figure $4(a)$ shows how the singular solution is approached for the rigid-wall case as $\alpha \rightarrow \bar{\alpha}_{r}$, and that $\tilde{\omega}_{r 1} \rightarrow \bar{\omega}$. The route for the rigid-wall case is unique because $c_{g}=c_{p}$.

We now need to consider which of the two routes, Route $\mathrm{A}$ and Route $\mathrm{B}$, are applicable to which respective situations, and this is discussed in the next two subsections. 

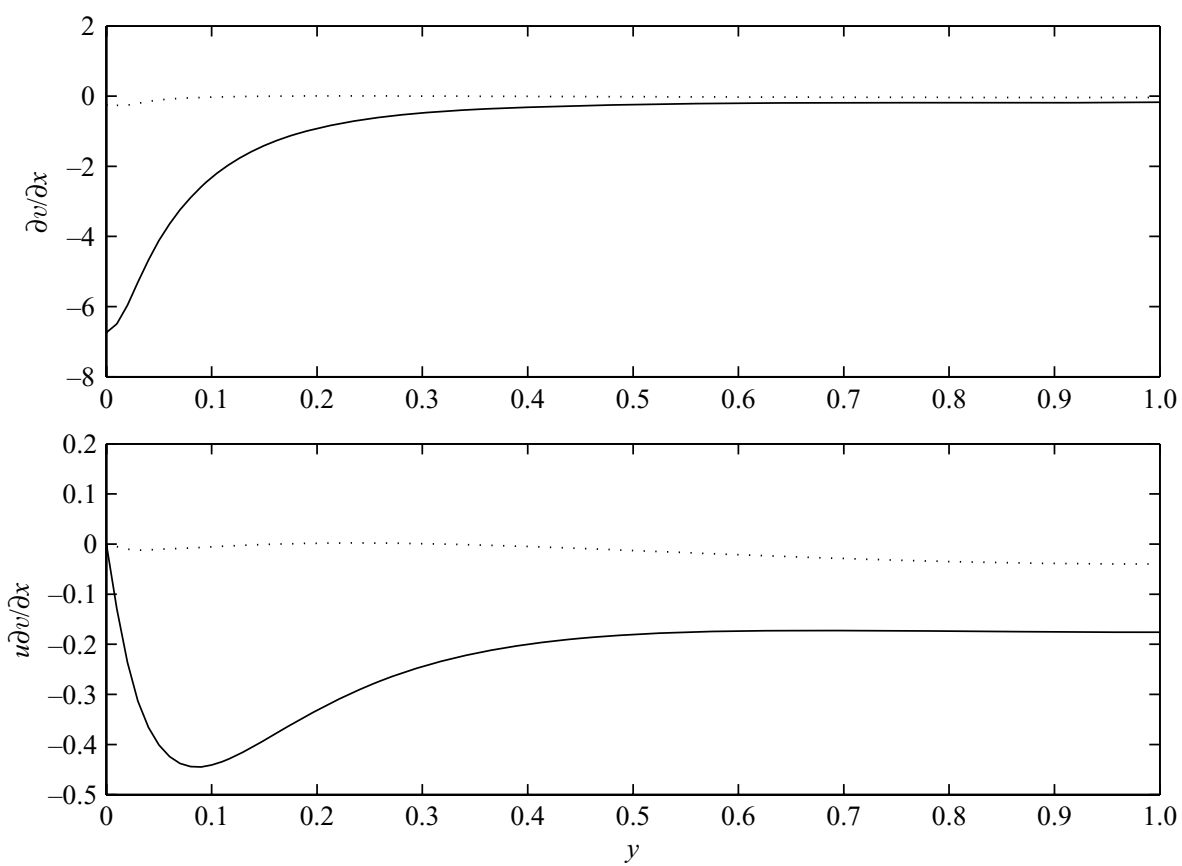

FIGURE 5. The variations of $\partial v / \partial x$ and $\bar{u} \partial v / \partial x$ with respect to $y$ evaluated from the numerical simulations for the driver given in (3.18) at $x=\ell / \sqrt{2}$ with $R e=12000, \bar{\omega}=0.24, \ell=0.125$ and $\hat{v}_{w}=\ell \sqrt{\pi}$.

\subsection{The wave driver at the leading-edge junction}

At the leading-edge junction the incoming wave is the rigid-side TS wave, and for this wave the wave driver behaves like a 'sink'. The outgoing wave is the compliant-side TS wave, but this need not necessarily be the only outgoing wave. For the time being we will assume that compliant-side TS wave is the only outgoing wave, and for this outgoing wave the wave driver is like a 'source'. The wave driver is therefore formed between the rigid-side TS wave and the compliant-side TS wave.

We now focus on the 'tuning in' process of the far-field compliant-side TS wave from the 'source' side of the wave driver. This process of tuning in is similar to that from any other (vibrating) source, for instance from a vibrating ribbon. Notionally it means that the far-field eigenvalue is selected from amongst the set of neighbouring physical eigenvalues. It is clear therefore that when a far-field wave is evolving from the 'source' side of the wave driver, Route A is the natural selection path in FourierLaplace space, and the operative group velocity is the regular group velocity $c_{g c}$. The real part of $c_{g c}$ and also that of $c_{g r}$ respectively for TSc (TS, compliant side) and TSr (TS, rigid side) are both positive. Therefore the TSr-TSc pair is compatible, and a wave driver forms at the leading-edge junction between this pair.

Now the question is can other far-field compliant waves tune in from the 'source' side of the wave driver formed between the TSr-TSc pair. At least theoretically we cannot discount the possibility for the case above cutoff. So, let us examine the other two waves on the compliant side that exist above cutoff, viz. the long wave $L$ and the vortical wave V. The long wave is nearly non-vortical; indeed its vorticity content is around $1 \%$ of that of the TSr wave. Hence, as per characteristic number 7 of a wave driver, listed in $\S 4.1$ earlier, the $\mathrm{L}$ wave cannot tune in from the wave driver at the 
leading edge. Next we consider the $\mathrm{V}$ wave. No doubt the $\mathrm{V}$ wave is strongly vortical, but both its regular group velocity and phase velocity have negative real parts, and the $\mathrm{V}$ wave, when formed, can propagate only in the upstream direction. Since the $\mathrm{V}$ wave cannot propagate upstream of the junction, i.e. along the rigid wall, it also does not tune in from the wave driver at the leading-edge junction. Actually both the $\mathrm{L}$ wave and the $\mathrm{V}$ wave are wall-based modes and are generated by mechanisms associated with wall displacement. How exactly the $\mathrm{L}$ wave and $\mathrm{V}$ wave are formed for the above cutoff case will be discussed in a later subsection.

In conclusion we may say that at the leading-edge junction a wave driver is formed between the TSr-TSc pair. The only outgoing wave from this wave driver is the compliant-side TS wave, viz. TSc, both for the cases below and above cutoff. Moreover, the selection route for approaching the singular solution in Fourier-Laplace space is Route A.

\subsection{The wave driver(s) at the trailing-edge junction}

At the trailing-edge junction there are two possible situations. First is for the below cutoff case, for which the incoming wave at the trailing-edge junction is the compliantside TS wave, viz. TSc. This wave goes across the junction and emerges as the downstream rigid-side TS wave, viz. TSr. A wave driver is formed between the TSc-TSr pair. For the incoming TSc wave this wave driver behaves like a 'sink'. The point to be noted here is that the incoming TSc already exists, and there is no tuning in process involved when this wave approaches the 'sink'. This subtle difference between the 'sink side' and the 'source side' of the wave driver should be carefully noted. From the source side the outgoing wave tunes in at the source and travels downstream. For the sink side, the incoming wave merely 'approaches' the wave driver, and there is no process of tuning in from neighbouring physical eigenvalues. Hence the singular solution in Fourier-Laplace space is approached through Route B, and the characteristic group velocity of the process is the pseudo group velocity $c_{p c}$. For the TSc wave, $c_{g c}$ and $c_{p c}$ are different, the difference increasing for above cutoff cases. Hence the TSr-TSc jump at the leading edge and the TSc-TSr jump at the trailing edge are not strictly reciprocal, especially above cutoff, although qualitatively the jumps look opposite of each other. Also, for the TSc wave, both $c_{g c}$ and $c_{p c}$ have positive real parts. Therefore, the TSc wave can emerge from the leading-edge wave driver and also can form the trailing-edge wave driver.

Now we consider the case above cutoff. Here, two waves, namely the TSc wave and the $\mathrm{L}$ wave, approach the trailing-edge junction. The $\mathrm{L}$ wave has very little vorticity, and therefore, as mentioned in characteristic number 7 in $\S 4.1$ earlier, the $\mathrm{L}$ wave does not form a wave driver at the trailing-edge junction. Both the TSc wave and the L wave are reflected at the trailing edge and reappear as the reflected $\mathrm{V}$ wave travelling upstream. This reflection is caused purely due to matching of the edge conditions at the junction of the compliant and rigid walls. Now the reflected $\mathrm{V}$ wave has a very interesting characteristic. Its regular group velocity $c_{g c}$ has a negative real part; therefore the $\mathrm{V}$ wave travels upstream along the compliant panel after being created at the trailing-edge junction. However the pseudo group velocity $c_{p}$ of the $\mathrm{V}$ wave has a positive real part; therefore, the $\mathrm{V}$ wave does also actually form a wave driver at the trailing edge. The outgoing TSr wave on the downstream rigid side is actually formed by the linear superposition of contributions from the TSc wave driver and also the $\mathrm{V}$ wave driver. This point is discussed in more detail later on in $\S 6$.

There is thus an important point for incoming waves approaching a junction. Each such approaching wave forms (when capable of forming) its own separate wave 
driver, because for the 'sink side' of the wave driver there are no neighbouring physical eigenvalues to tune in from. The outgoing wave, if it is a single wave, is actually formed by the superposition of each individual outgoing wave from each individual wave driver.

What remains now is to see the details of the mechanisms relating to how the wallbased waves, viz. the $\mathrm{L}$ wave and the $\mathrm{V}$ wave, are actually created. This is considered in the next subsection.

\subsection{The creation of wall-based waves}

Now we focus attention on how the $\mathrm{L}$ and $\mathrm{V}$ waves are generated in the compliant section for the above cutoff case. The amplitude of the TS wave over the compliant wall is calculated using (4.24). This is always in good agreement with the numerical simulations in Davies \& Carpenter (1997). Once the amplitude of the TS wave has been calculated, the amplitudes of the $\mathrm{V}$ and $\mathrm{L}$ waves can be determined by requiring the combination of all the eigenmodes present to satisfy the end conditions imposed on the compliant panel at the leading- and trailing-edge junctions. For pinned-jointed junction these are given by $(2.9 a, b)$; the corresponding conditions for clamped joints are given in $(2.10 a, b)$. Here we shall confine ourselves to the treatment for the pinnedend joints. Once we have used (4.24) to calculate the amplitude of the TS wave immediately downstream of the leading-edge junction, the $\mathrm{L}$ and $\mathrm{V}$ waves and the evanescent modes (see figure 3 ) can be determined from the four boundary conditions given in $(2.9 a, b)$. There is one complication. It turns out that there are four evanescent eigenmodes of the Orr-Sommerfeld compliant-wall eigensystem. (See Carpenter \& Morris 1990 who first identified these evanescent eigenmodes.) Of these four, only two are required, one at the leading-edge junction and one at the trailing-edge junction. Their selection has to be made with care. It seems reasonable to make the choice to correspond to minimum energy for the compliant wave system. Once this choice has been made by considering trial solutions for the four possible pairs of evanescent eigenmodes, we have four end conditions for determining the amplitudes of the four undetermined modes. This is demonstrated immediately below. The wall displacement comprises different components as follows:

$$
\eta=(\underbrace{A_{1} \mathrm{e}^{-\mathrm{i} \alpha_{1} x^{\prime}}}_{\text {TS }}+\underbrace{A_{2} \mathrm{e}^{-\mathrm{i} \alpha_{2}\left(x^{\prime}-l\right)}}_{\mathrm{V}}+\underbrace{A_{3} \mathrm{e}^{-\mathrm{i} \alpha_{3} x^{\prime}}}_{\mathrm{L}}+\underbrace{A_{4} \mathrm{e}^{-\mathrm{i} \alpha_{4} x^{\prime}}+A_{5} \mathrm{e}^{\mathrm{i} \alpha_{5}\left(x^{\prime}-l\right)}}_{\text {Evanescent }}) \mathrm{e}^{-\mathrm{i} \omega t},
$$

where $l=l_{2}-l_{1}$. The four end conditions $(2.9 a, b)$ can be arranged as simultaneous linear equations to determine the unknown amplitudes $A_{2}$ to $A_{5}$ in terms of the known TS amplitude $A_{1}$. Namely,

$$
\begin{aligned}
\eta=0 \quad \text { at } \quad x^{\prime}=0, & A_{2} \mathrm{e}^{-\mathrm{i} \alpha l}+A_{3}+A_{4}=-A_{1}, \\
\frac{\partial^{2} \eta}{\partial x^{2}} & =0 \quad \text { at } \quad x^{\prime}=0, \quad \alpha_{2}^{2} A_{2} \mathrm{e}^{-\mathrm{i} \alpha l}+\alpha_{3}^{2} A_{3}+\alpha_{4}^{2} A_{4}=-\alpha_{1}^{2} A_{1}, \\
\eta & =0 \quad \text { at } \quad x^{\prime}=l, \quad A_{2}+A_{3} \mathrm{e}^{-\alpha_{3} l}+A_{5}=-A_{1} \mathrm{e}^{-\alpha_{1} l}, \\
\frac{\partial^{2} \eta}{\partial x^{2}} & =0 \quad \text { at } \quad x^{\prime}=l, \quad \alpha_{2}^{2} A_{2}+\alpha_{3}^{2} A_{3} \mathrm{e}^{-\alpha_{3} l}+\alpha_{5}^{2} A_{5}=-\alpha_{1}^{2} A_{1} \mathrm{e}^{-\alpha_{1} l} .
\end{aligned}
$$

Using the above procedure, very good agreement is obtained between the numerical simulations in Davies \& Carpenter (1997) and the present results.

Next comes the question of smoothening the TSc wave at the two junctions for the below cutoff case. It is a routine matter to use evanescent modes (which also exist below cutoff), two at the leading-edge junction and two at the trailing-edge 
junction. The two at the leading-edge junction must damp downstream, and the two at the trailing-edge junction must damp upstream. However, the proportion of the evanescent modes will be different respectively for hinged-joint and clamped-joint cases. This smoothening process at the edges is of cosmetic value only for the below cutoff case, quite unlike for the above cutoff case. For the latter the matching of edge conditions is a must in order to get the entire compliant wave system, especially the amplitudes of the $\mathrm{L}$ wave and $\mathrm{V}$ wave. We have presented the results for the below cutoff case without any smoothening at the edges to highlight that the jumps are no different with either clamped or hinged joints, which is also confirmed by numerical simulations.

\section{Determining the driver function $\mathscr{F}(y)$}

In this section we describe how the form of the driver function $\mathscr{F}(y)$ may be educed from a local solution of the linearized Navier-Stokes equation (2.2) in the region of the junction. For this problem there is no role of $\alpha$. However, we do remember that $\bar{\omega}$, along with the rigid-wall far-field eigenvalue $\bar{\alpha}_{r}$, is located at a point in the $(\alpha, R e)$-plane that corresponds to the upper branch of the neutral curve. We can therefore consider $\bar{\omega}$ to be either constant or varying along the upper branch as $\sim O\left(R e^{-3 / 11}\right)$. The subsequent analysis addresses both possibilities. Since $\alpha$ has no role in the subsequent analysis, we consider the function $f=f(x, y)$ given from (2.2). Further we remember from (4.3) that the strength of the driver is $C \sim O\left(R^{-2 / 3}\right)$. Also the form given by (3.3) suggests that the width of the near-field region (of which the driver is an idealized discretization) should have a width $\epsilon$ of the order of $\sim O\left(R e^{-1 / 3}\right)$. Therefore we introduce an $x$-wise scale for the near field at the junction as $\epsilon=\left(R e^{-1 / 3}\right)$. Moreover, we define the perturbation vorticity as $\zeta$ in this near-field region. An alternative form of (2.2) in terms of $\zeta$ is given below:

$$
\begin{gathered}
\mathrm{i} \bar{\omega} \zeta+\bar{u} \frac{\partial \zeta}{\partial x}-\bar{u}^{\prime \prime} \frac{\partial f}{\partial x}-\frac{1}{R e} \nabla^{2} \zeta=0, \\
\nabla^{2} f=-\zeta .
\end{gathered}
$$

We conceive of the asymptotic structure of the flow field in the vicinity of the junction as consisting of an outer region having $O(1)$ thickness with an inner viscous layer of thickness $O\left(\epsilon_{i}\right)$ adjoining the wall; $\epsilon_{i}$ has to be determined by an asymptotic analysis, where the generic subscript ' $i$ ' is used for the inner region.

In the outer region the flow variables can be approximated by the asymptotic expansions

$$
\zeta=\epsilon^{-2} \zeta_{0}(\xi, y)+\cdots, \quad f=f_{0}(\xi, y)+\cdots,
$$

where $\xi=x / \epsilon$. An order-of-magnitude analysis of (5.1) gives

$$
\underbrace{\mathrm{i} \bar{\omega} \zeta}_{O\left(\epsilon^{-2}\right)=O\left(R e^{2 / 3}\right)}+\underbrace{\bar{u} \frac{\partial \zeta}{\partial x}}_{O\left(1 / \epsilon^{3}\right)=O(R e)}-\underbrace{\bar{u}^{\prime \prime} \frac{\partial f}{\partial x}}_{O(1 / \epsilon)=O\left(R e^{1 / 3}\right)}-\underbrace{\frac{1}{R e} \frac{\partial^{2} \zeta}{\partial x^{2}}}_{O\left(1 / R e \epsilon^{4}\right)=O\left(R e^{1 / 3}\right)}-\underbrace{\frac{1}{R e} \frac{\partial^{2} \zeta}{\partial y^{2}}}_{O\left(1 /\left(R e \epsilon^{2}\right)=O\left(R e^{-1 / 3}\right)\right.}=0 .
$$

So the second term is dominant. Therefore (5.4) reduces to $\partial \zeta_{0} / \partial \xi=0$ with the general solution

$$
\zeta_{0}=\zeta_{0}(y)
$$


Equation $(5.3 a, b)$ is then substituted into $(5.2)$ to obtain at largest order

$$
\frac{\partial^{2} f_{0}}{\partial \xi^{2}}=-\zeta_{0}(y)=F_{0}(y),
$$

where $F_{0}$ will eventually be related to $\mathscr{F}(y)$, and a separate symbol $F_{0}$ is therefore introduced in place of $\zeta_{0}$. The general solution of (4.31) is given by

$$
f_{0}(\xi, y)=F_{0}(y) X(\xi)+F_{1}(y) \xi+F_{2}(y) .
$$

Since the near field is a narrow viscous region eventually merging into the far field on either side of the junction, a symmetric form for $X(\xi)$, around $\xi=0$, is suggestive. Also $F_{1}$ and $F_{2}$ are general integration functions of $y$, but are of little consequence here since they do not contribute to the driver function $\bar{u} \partial^{2} f /\left.\partial \xi^{2}\right|_{\xi=0}$, and we may put $F_{1}, F_{2}=0$.

Since the driver function needs to be educed from the limit $\xi \rightarrow 0$, we confine ourselves to within the neighbourhood of $\xi=0$. Thus $X(\xi)$ is put

$$
X(\xi)=1-\frac{\xi^{2}}{2} \text {. }
$$

The outer solution for $f_{0}$ is called $f_{o 0}$, with the generic subscript ' $o$ ' for the outer region. Since all the far-field eigenfunctions for both compliant and rigid sides are symmetric with respect to the channel centreline, it is logical to assume that $f_{0}$ will be symmetric with respect to the centreline. Hence simple polynomial functions, symmetric about the channel centreline, are admissible candidates. A set of admissible forms could be given as

$$
F_{o 0}=a_{0}+u_{n}(y), \quad u_{n}(y)=\left[1-(1-y)^{2 n}\right],
$$

with $n$ assuming any one of the integer values $n=1,2,3, \ldots$ Note that $u_{n}(0)=0$ for all $n$. There is some limitation on the largest value of $n$ admissible; also, it will be shown later that low values of $n$ are appropriate. Both these aspects will be seen when the inner-wall viscous solution is considered, which is discussed next.

In the inner region of thickness $O\left(\epsilon_{i}\right)$ the stretched variable $y_{i}=y / \epsilon_{i}$ is introduced. $\epsilon_{i}$ is unknown a priori, but it must be such that viscous effects are significant near the wall. The dependent variables in the inner region are approximated by an asymptotic expansion of the form

$$
f_{i}=f_{i 0}\left(\xi, y_{i}\right)+O\left(\epsilon_{i}\right)+\cdots .
$$

The corresponding expression for vorticity $\zeta_{i 0}$ is interesting. It is given as

$$
\zeta_{i 0}=\epsilon^{-2} \frac{\partial^{2} f_{i 0}}{\partial \xi^{2}}+\epsilon_{i}^{-2} \frac{\partial^{2} f_{i 0}}{\partial y_{i}^{2}} .
$$

So in (5.1), the ordering of terms has to be done depending on whether $\epsilon \sim o\left(\epsilon_{i}\right)$ or $\epsilon_{i} \sim o(\epsilon)$. First we will assume that $\epsilon_{i} \sim o(\epsilon)$. Further we assume that the form for $X(\xi)$, as given in (5.8), carries over also to the inner-wall region. From (5.1) and (5.4), read in conjunction with (5.8), we have the following equations respectively for $\zeta_{i 0}$ and $f_{i 0}$ at the largest order, viz. $\sim O\left(\epsilon_{i}^{-2}\right)$ :

$$
\frac{1}{R e \epsilon_{i}^{2}} \frac{\partial^{2} \zeta_{i 0}}{\partial y_{i}^{2}}-\mathrm{i} \bar{\omega} \zeta_{i 0}=0 \quad \text { or } \quad \frac{1}{R e \epsilon_{i}^{2}} \frac{\partial^{4} f_{i 0}}{\partial y_{i}^{4}}-\mathrm{i} \bar{\omega} \frac{\partial^{2} f_{i 0}}{\partial y_{i}^{2}}=0 .
$$

For topical interest we also consider the case where $\epsilon \sim o\left(\epsilon_{i}\right)$. (This might actually be the case if $\bar{\omega}$ is considered along the lower branch of the neutral of the neutral 
curve.) For $\epsilon \sim o\left(\epsilon_{i}\right)$, (5.1) gives the following equation for $f_{i 0}$ at the largest order, viz. $\sim O\left(\epsilon^{-2}\right)$ :

$$
\frac{2}{R e \epsilon_{i}^{2}} \frac{\partial^{4} f_{i 0}}{\partial \xi^{2} \partial y_{i}^{2}}-\mathrm{i} \bar{\omega} \frac{\partial^{2} f_{i 0}}{\partial \xi^{2}}=0 .
$$

The above equation is only at second order in $y_{i}$ and the solution for $f_{i 0}$ is unable to match the two wall boundary conditions, viz. $f_{i 0}(\xi, 0), f_{i 0}^{\prime}(\xi, 0)=0$, and also boundedness in the outer region. Here prime denotes partial derivative with respect to $y_{i}$. In fact with $f_{i 0}(\xi, 0), f_{i 0}^{\prime}(\xi, 0)=0$, only the trivial solution exists. So, therefore, we need to go to the next higher order, viz. $\sim O\left(\epsilon_{i}^{-2}\right)$, which gives $(5.11 a, b)$.

In $(5.11 a, b), \bar{\omega}$ may be considered either to be a constant or to be along the upper branch of the neutral curve, for which $\bar{\omega} \sim O\left(R e^{-3 / 11}\right)$. Thus we have two alternative versions of $(5.11 a, b)$. For constant $\bar{\omega}$ we have

$$
\epsilon_{i}=R e^{-1 / 2}
$$

which gives

$$
\frac{\partial^{2} \zeta_{i 0}}{\partial y_{i}^{2}}-\mathrm{i} \bar{\omega} \zeta_{i 0}=0 \quad \text { or } \quad \frac{\partial^{4} f_{i 0}}{\partial y_{i}^{4}}-\mathrm{i} \bar{\omega} \frac{\partial^{2} f_{i 0}}{\partial y_{i}^{2}}=0 .
$$

For $\bar{\omega} \sim O\left(R^{-3 / 11}\right)$, along the upper branch of the neutral curve, we introduce the current Reynolds number $R e=R_{0}$, and express $\omega$ at any $R e$ as follows:

$$
\omega=\bar{\omega} r^{-3 / 11}, \quad r=\frac{R e}{R_{0}} .
$$

If we now put

$$
\epsilon_{i}=R_{0}^{-1 / 2} r^{-4 / 11}
$$

we actually do get back the same set of equations as in $(5.13 a, b)$. So, we note that in either case we need to contend with $(5.13 a, b)$, only that $\epsilon_{i}$ is different. In the same manner one could also determine a suitable $\epsilon_{i}$ with $\omega$ corresponding to the lower branch of the neutral curve and still retain the same forms as in $(5.13 a, b)$.

The solution for $(5.13 b)$, irrespective of choice of $\epsilon_{i}$, is what we now need to look for. Remembering (5.7) and (5.8), we express $f_{i 0}$ as follows:

$$
\begin{gathered}
f_{i 0}=F_{i 0}\left(y_{i}\right)\left(1-\frac{\xi^{2}}{2}\right), \\
F_{i 0}\left(y_{i}\right)=C_{1}+C_{2} y_{i}+C_{3} \mathrm{e}^{-\sigma y_{i}}+C_{4} \mathrm{e}^{\sigma y_{i}}, \quad \sigma=(1+\mathrm{i}) \sqrt{\frac{\bar{\omega}}{2}} .
\end{gathered}
$$

The first two terms on the right-hand side of (5.16b), $C_{1}$ and $C_{2} y_{i}$, are consistent with the polynomial form of the outer solution, i.e. $(5.9 a, b)$, and these terms are recovered from the outer solution when the outer solution is expressed in inner form. As shown later below, implementing this last step gives the order of the term $a_{0}$, in $(5.9 a)$, as $a_{0} \sim C_{1} \sim O\left(\epsilon_{i}\right)$. Moreover, $C_{4}=0$ since $\mathrm{e}^{\sigma y_{i}}$ grows away from the wall.

Before attempting to obtain a composite solution, comprising inner and outer solutions, we look at the boundary conditions for $f_{i 0}$. The boundary conditions at the wall are

$$
\frac{\partial f_{i 0}}{\partial \xi}=\frac{\partial f_{i 0}}{\partial y_{i}}=0, \quad y_{i}=0,
$$

which correspond to the normal and tangential velocities being zero at the wall. We note that $(5.17 a)$ has to be valid for all $\xi$ in the domain of $\xi$, viz. $|\xi|<1$. This 
translates to the wall boundary conditions being equivalent to

$$
f_{i 0}=\frac{\partial f_{i 0}}{\partial y_{i}}=0, \quad y_{i}=0,
$$

which is tantamount to saying that

$$
F_{i 0}\left(y_{i}\right)=F_{i 0}^{\prime}\left(y_{i}\right)=0, \text { for } y_{i}=0 .
$$

The composite solution $F_{0}$, comprising $F_{o 0}, F_{i 0}$ after introducing a quantity $\chi=\sigma / \epsilon_{i}$, can be written as

$$
F_{0}(y)=u_{n}(y)-\frac{u_{n}^{\prime}(0)}{\chi}\left(1-\mathrm{e}^{-\chi y}\right) .
$$

The constant term, corresponding to $C_{1}$, appears as $u_{n}^{\prime}(0) / \chi$ which is $\sim O\left(\epsilon_{i}\right)$, with $\chi \sim O\left(\epsilon_{i}^{-1}\right)$. This, however, sets a limitation of the order of largeness of $n$ that is admissible. We may see from $(5.9 b)$ that $u_{n}^{\prime}(0) \sim O(n)$. Hence, for $(5.19)$ to be consistent, it is necessary that $n$ be smaller than $\epsilon_{i}^{-1}$; in other words $n \sim o\left(\epsilon_{i}^{-1}\right)$.

The form given by (5.19) is versatile. It allows not only a range of outer functions $u_{n}(y)$, but also a range of forms for $\epsilon_{i}$, e.g. (5.12) and (5.15). Finally, we therefore obtain the driver function as

$$
\mathscr{F}(y)=\bar{u} F_{0}(y) .
$$

We now recall that the driver function is used only in integral expressions, like

$$
I_{2}=\int_{0}^{1} \mathscr{F}(y) \theta \mathrm{d} y .
$$

This being the case the viscous correction in the form of $F_{0}(y)$, vide (5.19), is only of cosmetic value since neglecting the viscous correction at most results in an error $\sim O\left(\epsilon_{i}\right)$ in the value of the integrals. Hence, for all practical purposes it suffices to consider only the outer inviscid form of the driver function, that is

$$
\mathscr{F}(y)=\bar{u} u_{n}(y) .
$$

Further, we look at the form for $u_{n}(y)$ given in $(5.9 b)$ and evaluate the integral $I_{n}$ given as

$$
I_{n}=\int_{0}^{1} u_{n}(y) \mathrm{d} y=1-\frac{1}{2 n+1}
$$

Next we observe

$$
\frac{\mathrm{d} I_{n}}{\mathrm{~d} n}=\frac{2}{(2 n+1)^{2}} \sim O\left(n^{-2}\right) .
$$

Hence, as $n$ becomes large, the rate of increase of $I_{n}$ actually decreases rapidly, as $\sim O\left(n^{-2}\right)$. Now, for $n=1, u_{1}(y)$ actually is $u_{1}(y)=\bar{u}(y)$. Also for large $n, u_{n}(y)$ becomes more and more flat in the centreline region, and for $n \rightarrow \infty$, the function $u_{n}(y)$ is virtually a constant and may be put equal to 1 . This being the case the driver function is well represented within the range

$$
\mathscr{F}(y)=\bar{u} \bar{u}, \quad n=1 ; \quad \mathscr{F}(y)=\bar{u}, \quad n=\infty .
$$

In our numerical work we actually investigated both the forms $(5.25 a, b)$. Expectedly the results for jump in amplitude were not very different using either. Mainly this is because of (5.24), which shows that contribution to the integral expressions rapidly decreases as $n$ increases. 

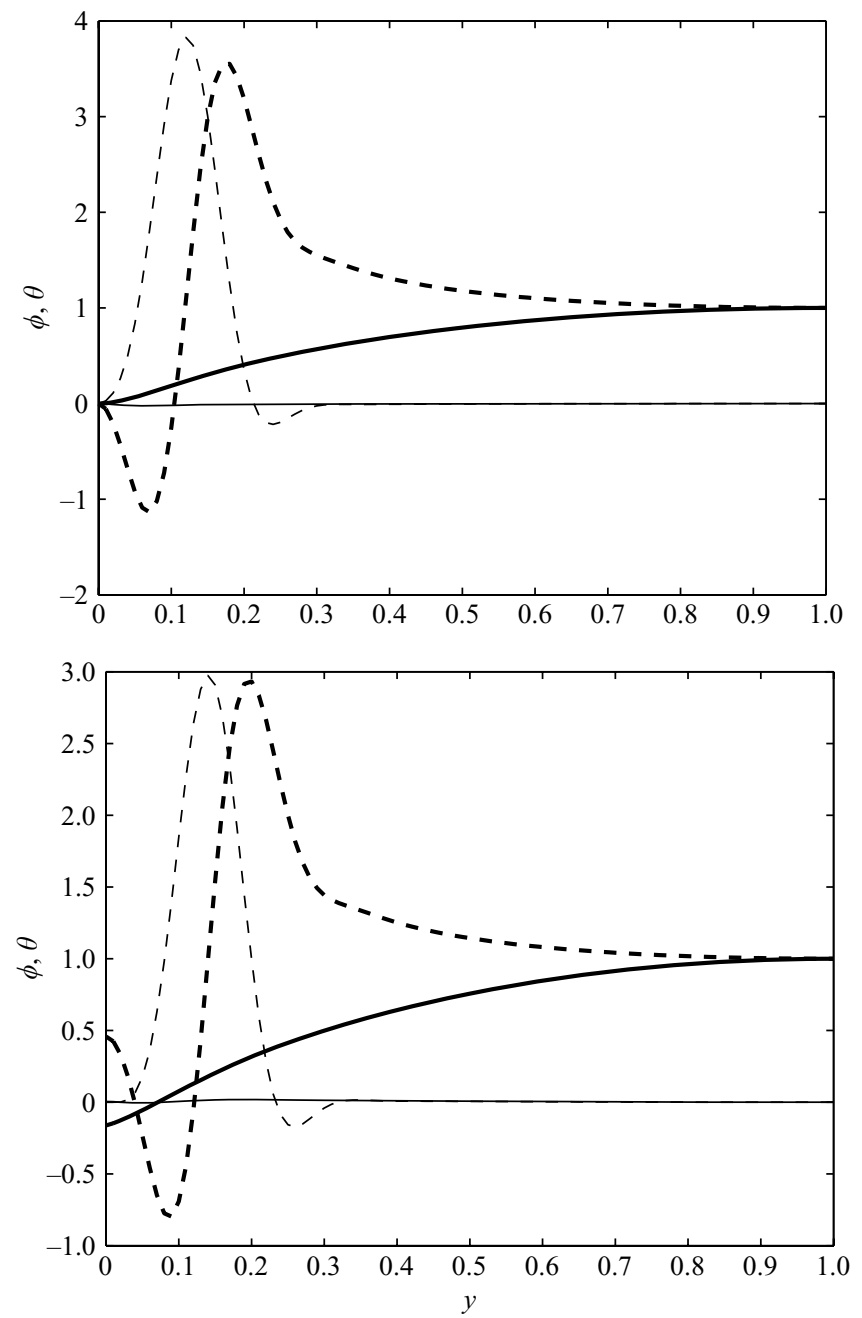

FIGURE 6. Eigenfunction $\phi$ (unbroken lines) and adjoint eigenfunction $\theta$ (broken lines), for the TS mode over the rigid wall (upper figure) and over the compliant wall (lower figure). The real and imaginary parts are denoted by the thick and thin lines respectively. $R e=12000$ and $\omega=0.24$. The compliant wall properties are given in (6.1).

\section{Results and discussion}

\subsection{General results}

The actual numerical results are presented from figure 6 onwards. The eigenfunction $\phi$ and the adjoint eigenfunction $\theta$ are given for the TS waves, both rigid and compliant, in figure 6. Figure 7 gives the vorticity function $g=\phi^{\prime \prime}-\alpha^{2} \phi$ for the same two cases. Note that $g$ is defined slightly differently from (3.5), but this is only a difference in normalization. Figure 8 shows the $\phi$ and $\theta$ functions corresponding to the $\mathrm{V}$ wave and $\mathrm{L}$ wave. And figure 9 gives the $g$ function for the $\mathrm{L}$ wave and $\mathrm{V}$ wave. The most noteworthy features in these figures is that the TS waves, both rigid and compliant, and also the $\mathrm{V}$ waves are strongly vortical, as may be seen from the respective $g$ functions. However, the $\mathrm{L}$ wave is virtually non-vortical by comparison, as may be seen from the corresponding $g$ function for the $\mathrm{L}$ wave in figure 9. 

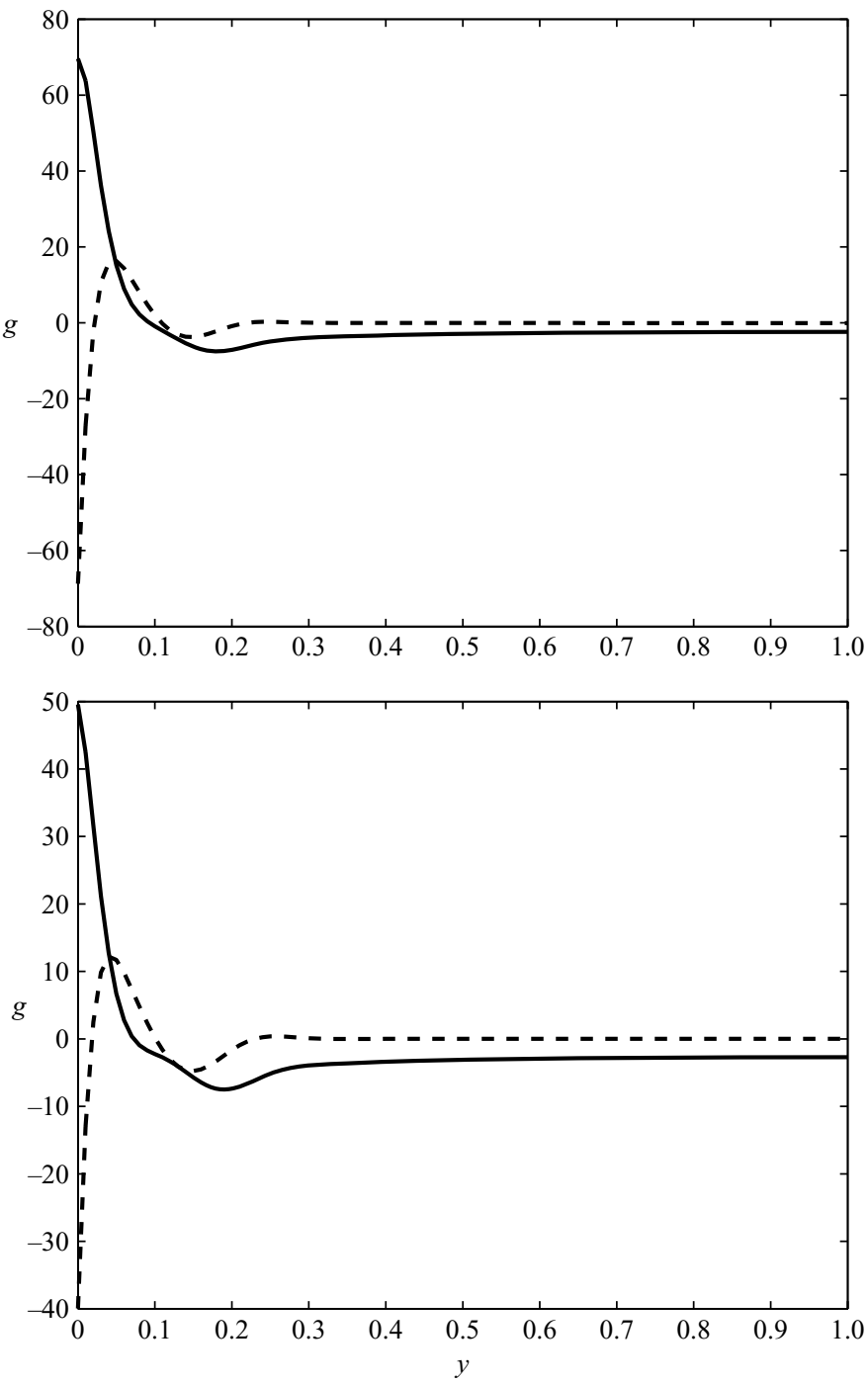

FIGURE 7. Vorticity functions $g=\phi^{\prime \prime}-\alpha^{2} \phi$ for the TS mode over the rigid wall (upper figure), and over the compliant wall (lower figure). The notation and parameters are the same as for figure 6 .

Table 1 gives the eigenvalues $\alpha$ for the various modes for $R e=12000$ and $\bar{\omega}=0.24$. Table 2 gives the complex phase velocity $c$, group velocity $c_{g}$ and the pseudo group velocity $c_{p}$ for the various modes. Table 2 shows that the real parts of $c, c_{g}$ and $c_{p}$ are all positive for the TSr wave and also for the TSc waves, for both below and above cutoff cases. Also $c_{g}$ and $c_{p}$ are the same for the TSr wave. The L wave depicts anomalous behaviour, in that the real part of its phase velocity $c$ is negative, but the real part of its group velocity $c_{g}$ is positive. The $\mathrm{L}$ wave therefore propagates downstream, when formed at the leading edge, despite the real part of its phase velocity being negative. Also, for the $\mathrm{V}$ wave the real parts of its phase velocity $c$ and group velocity $c_{g}$ are both negative. Hence the $\mathrm{V}$ wave is formed at the trailing-edge junction and propagates upstream along the compliant panel. However, the interesting 

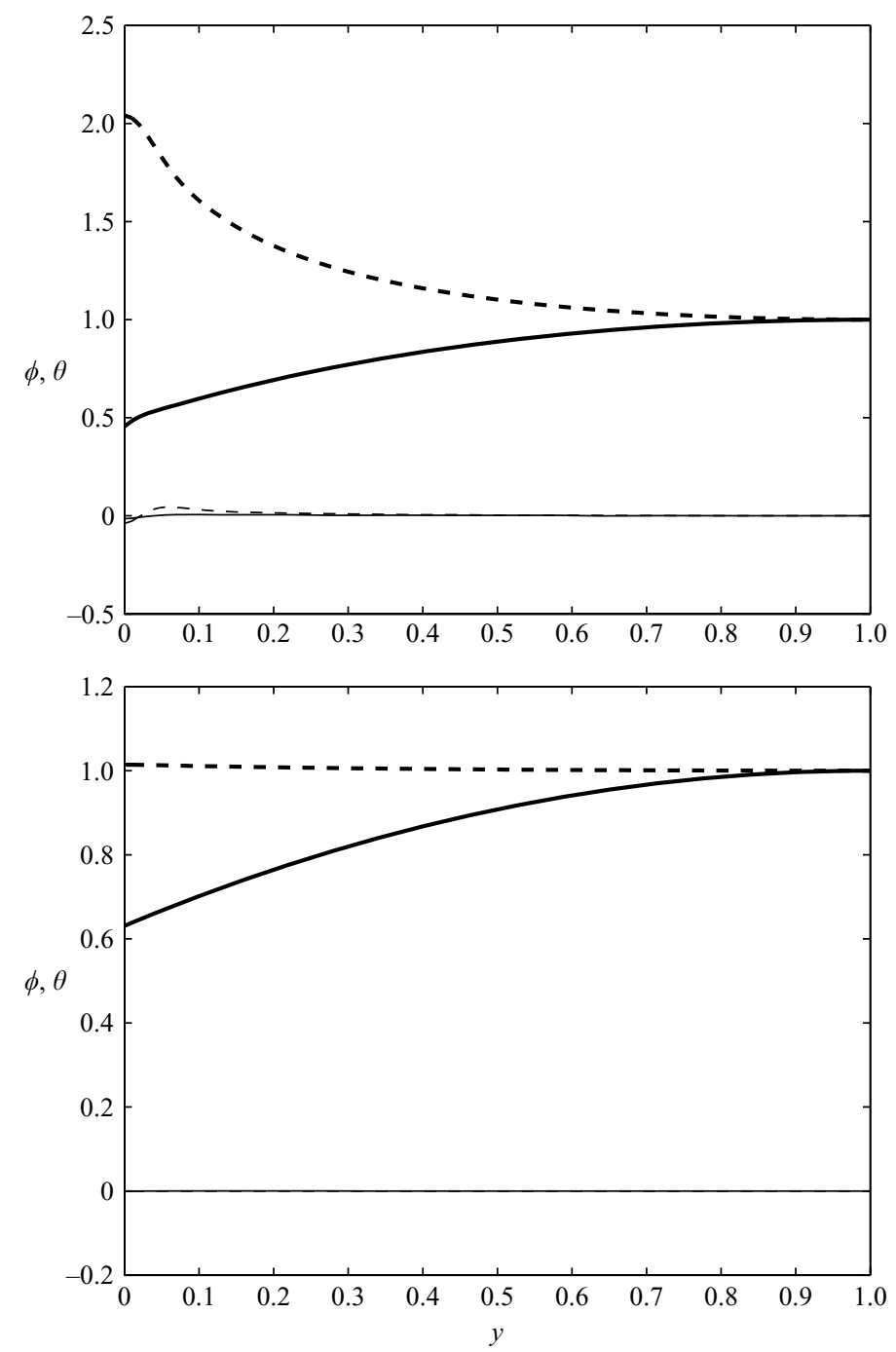

FIGURE 8. Eigenfunction $\phi$ (unbroken lines) and adjoint eigenfunction $\theta$ (broken lines) for the $\mathrm{V}$ mode (upper figure) and and the L mode (lower figure) The real and imaginary parts are denoted by the thick and thin lines respectively. $R e=12000$ and $\bar{\omega}=0.24$.

point about the $\mathrm{V}$ wave is that the real part of its pseudo group velocity $c_{p}$ is positive. Hence, as discussed in $\S 4.5$ earlier, the $\mathrm{V}$ wave is actually capable of producing a wave driver at the trailing edge from which the downstream rigid-side TSr wave emerges. This point is discussed in more detail later on.

\subsection{Results for TS frequency below cutoff}

For this case, that is the same as the one considered in Davies \& Carpenter (1997), we set $R e=12000$ which is about twice the critical value for a rigid-wall channel. The wall parameters are

$$
m=\frac{1}{3}, \quad d=0, \quad B=1.92 \times 10^{7}, \quad K=4 B .
$$



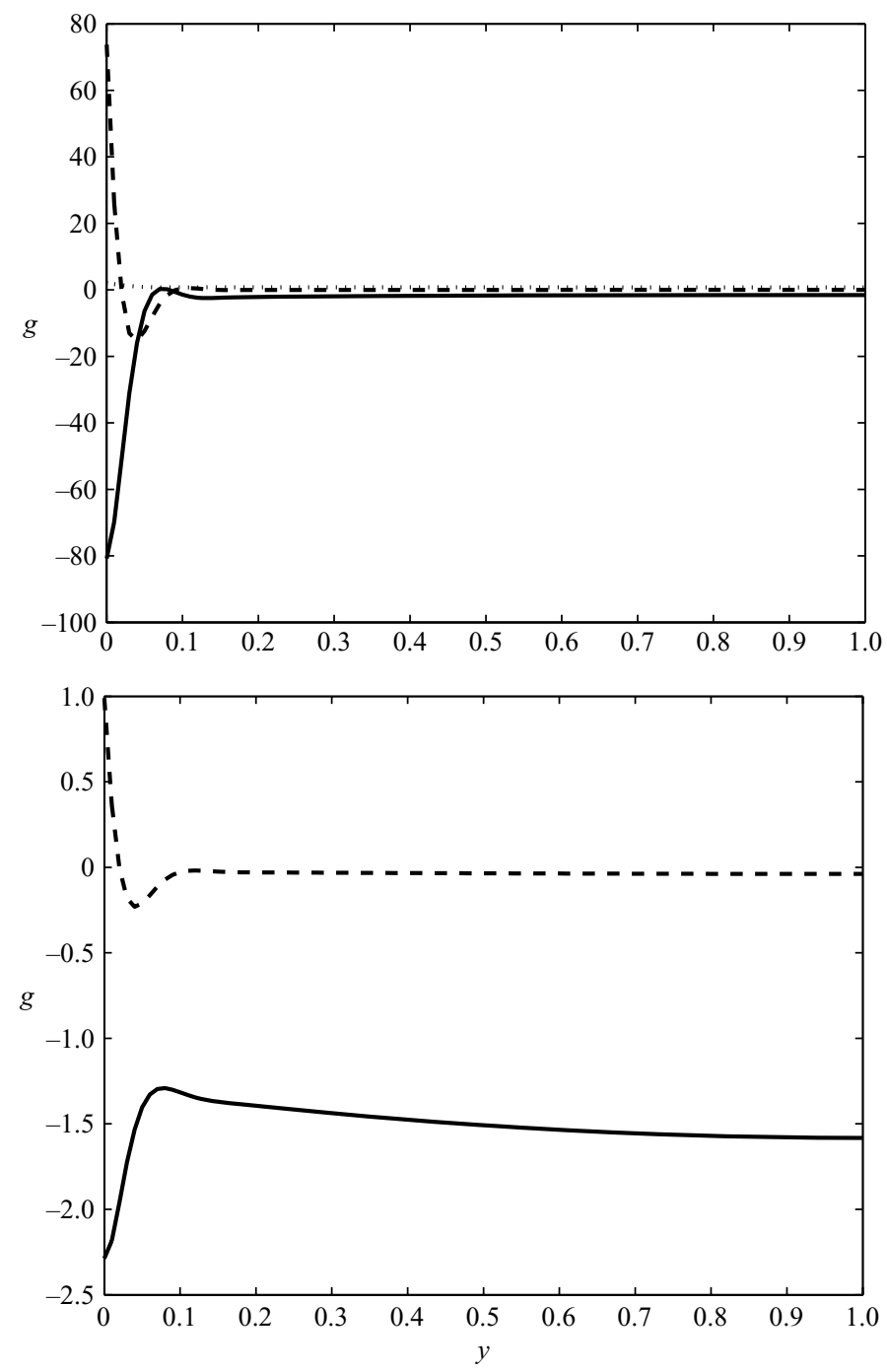

FIGURE 9. Vorticity functions, $g=\phi^{\prime \prime}-\alpha^{2} \phi$, for the V mode (upper figure) and the L mode (lower figure). The notation and parameters are the same as for figure 8 .

These property values are quite similar to those attributed by Carpenter \& Garrad (1985) to the original Kramer (1960) compliant coatings.

The non-dimensional TS frequency chosen and the corresponding eigenvalues are

$$
\bar{\omega}=0.24, \quad \bar{\alpha}_{r}=1.03-0.009 \mathrm{i} \quad \text { (rigid), } \quad \bar{\alpha}_{c}=0.940+0.0109 \mathrm{i} \quad \text { (compliant). }
$$

This corresponds to a case where the TS wave is amplified over the rigid surface but attenuated over the compliant wall. Two forms of the driver function $\mathscr{F}$ are used. These are respectively given by $\mathscr{F}(y)=\bar{u} \bar{u}$ and $\mathscr{F}(y)=\bar{u}$, as discussed in $\S 5$ and as given by $(5.25 a, b)$. The values for the jumps in wave amplitude at the leading- and trailing-edge junctions are given in table 3 for the two forms of the wave driver function. Also given in table 3 are comparable results from numerical simulations carried out in a similar manner to that described in Davies \& Carpenter (1997). 


\begin{tabular}{lc}
\multicolumn{1}{c}{ Mode } & Wavenumber $(\bar{\alpha})$ \\
Rigid TS (TSr) & $(1.0317,-0.0093)$ \\
Compliant TS (TSc BC) & $(0.9395,0.0109)$ \\
Compliant TS (TSc AC) & $(0.9207,0.0212)$ \\
Vortical (V) & $(-0.8332,-0.0138)$ \\
Longwave (L) & $(-0.1459,0.00013)$ \\
Evanescent (EV1) & $(0.6835,0.3144)$ \\
Evanescent (EV2) & $(-0.3788,-1.0485)$
\end{tabular}

TABLE 1. Eigenvalues corresponding to the various eigenmodes below and above cutoff $(R e=12000, \bar{\omega}=0.24)$. Note: $\mathrm{BC}$, below cutoff; AC, above cutoff.

$\begin{array}{lccc}\quad c & c & c_{g} & c_{p} \\ \text { TSr } & (0.233,0.002) & (0.325,-0.042) & (0.325,-0.042) \\ \text { TSc (BC) } & (0.255,-0.003) & (0.375,-0.110) & (0.395,-0.099) \\ \text { TSc (AC) } & (0.261,-0.006) & 0.285,-0.068) & (0.408,-0.113) \\ \text { V } & (-0.288,0.005) & (-0.783,-0.033) & (0.117,0.022) \\ \text { L } & (-1.645,-0.939) & (0.535,0.002) & (-1.477,-0.002)\end{array}$

TABLE 2. Values of integral phase speed $(c)$, group velocity $\left(c_{g}\right)$ and pseudo group velocity $\left(c_{p}\right)$. Note: BC, below cutoff; AC, above cutoff.

$\begin{array}{ccc}\text { Theory } & \text { Theory } & \\ \text { Case } & \mathscr{F}(y)=\bar{u}^{2} & \mathscr{F}(y)=\bar{u} \quad \text { Numerical simulation }\end{array}$

$\begin{array}{lccc} & & 1.22 & 1.22 \\ \text { TSc/TSr (BC LEJ HJ) } & 1.24 & 1.22 & 1.24 \\ \text { TSc/TSr (BC LEJ CJ) } & 1.24 & 1.17 & 1.19 \\ \text { TSc/TSr (BC TEJ HJ) } & 1.19 & 1.17 & 1.18 \\ \text { TSc/TSr (BC TEJ CJ) } & 1.19 & 1.74 & 1.67 \\ \text { TSc/TSr (AC LEJ HJ) } & 1.78 & 1.21 & - \\ \text { TSc/TSr (AC LEJ HJ) } & 1.23 & 1.53 & 2.59 \\ \text { L/TSr (LEJ HJ) } & 1.62 & 1.53 & 2.9 \\ \text { L/TSr (TEJ HJ) } & 1.62 & 2.69 & \end{array}$

TABLE 3. Absolute magnitudes of the ratio of the amplitudes of specified mode to rigid TS wave. Note: BC, below cutoff; AC, above cutoff; LEJ, leading-edge junction; TEJ, trailing-edge junction; HJ, hinjed joint; CJ, clamped joint.

In the numerical simulations the far-field solutions were extrapolated back to the joints in order to determine the respective amplitudes $a$ from which the jumps were determined.

Several points emerge from the results given in table 3 for the below cutoff case. First, we see the results that are given for both hinged and clamped joints. According to our theory the results should be identical in the two cases. It can be seen that the results of the numerical simulations in the two cases do indeed differ very little, much less than the difference in jump values between the leading- and trailing-edge junctions. Second, there is very close agreement between the theoretical and numerical results. Third, we note that there is near-reciprocity between the jumps at the leadingand trailing-edge junctions. Precise reciprocity would be found if the pseudo group velocity $c_{p}$ and the regular group velocity $c_{g}$ were the same for the TSc wave. As 


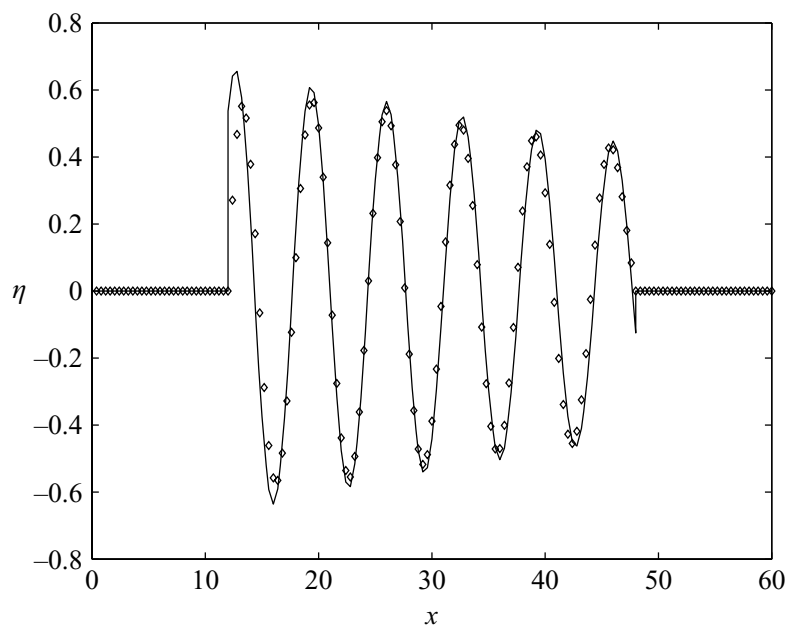

FIGURE 10. Streamwise variation of the wall displacement for the case when the incident wave is below the cutoff frequency of the compliant panel. The solid line denotes the results of our present theory and the data points represent the results of the numerical simulation of Davies \& Carpenter (1997). The leading and trailing edges of the panel are at $x=\ell_{1}=12$ and $x=\ell_{2}=48$ respectively; $R e=12000$ and $\bar{\omega}=0.24$; the compliant-wall properties and wavenumbers are given in $(6.1 a, b)$.

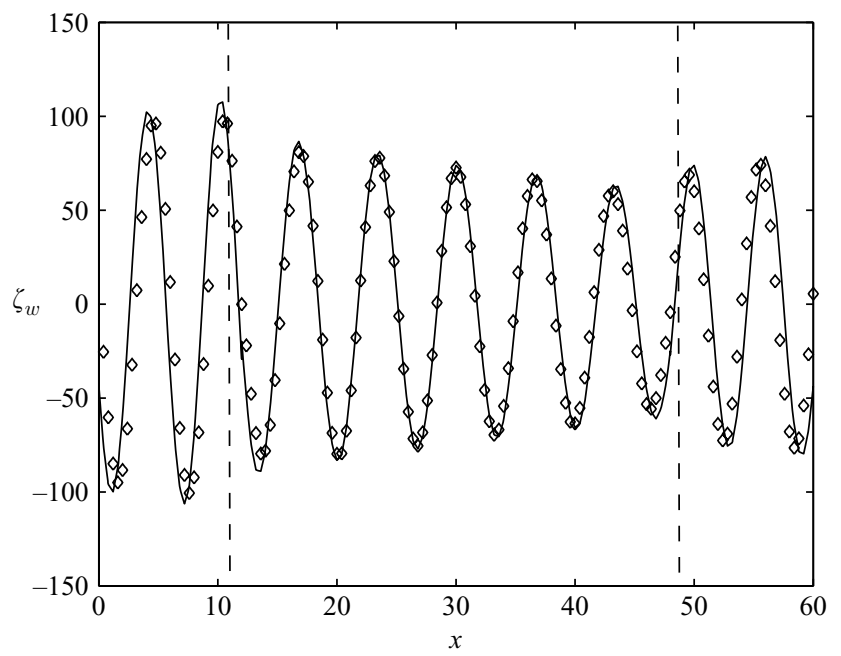

FIGURE 11. Streamwise variation of the perturbation vorticity $\zeta_{w}$, evaluated at the wall, for the same case as in figure 10. Other features as in figure 10.

table 2 shows, the difference between $c_{p}$ and $c_{g}$ for the TSc wave is not very large for the below cutoff case. However, the lack of precise reciprocity, as seen in the results of the numerical simulations, is captured well by the theory, as may be seen in table 3. Finally, it can be seen in table 3 that there is little difference between the results for the two types of the wave driver function, showing that the precise form of the wave driver function is relatively unimportant.

The wall displacement $\eta$ and the wall vorticity $\zeta_{w}$ are plotted respectively in figures 10 and 11. Also plotted as data points are the results obtained by the 


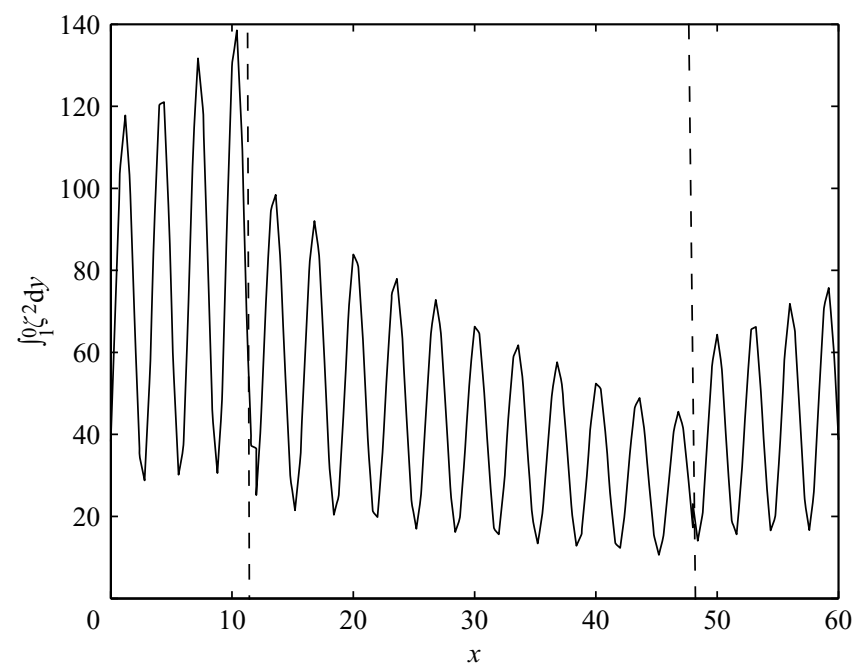

FIGURE 12. Streamwise variation of the perturbation vorticity integral for the same case as in figure 10. Other features as in figure 10 .

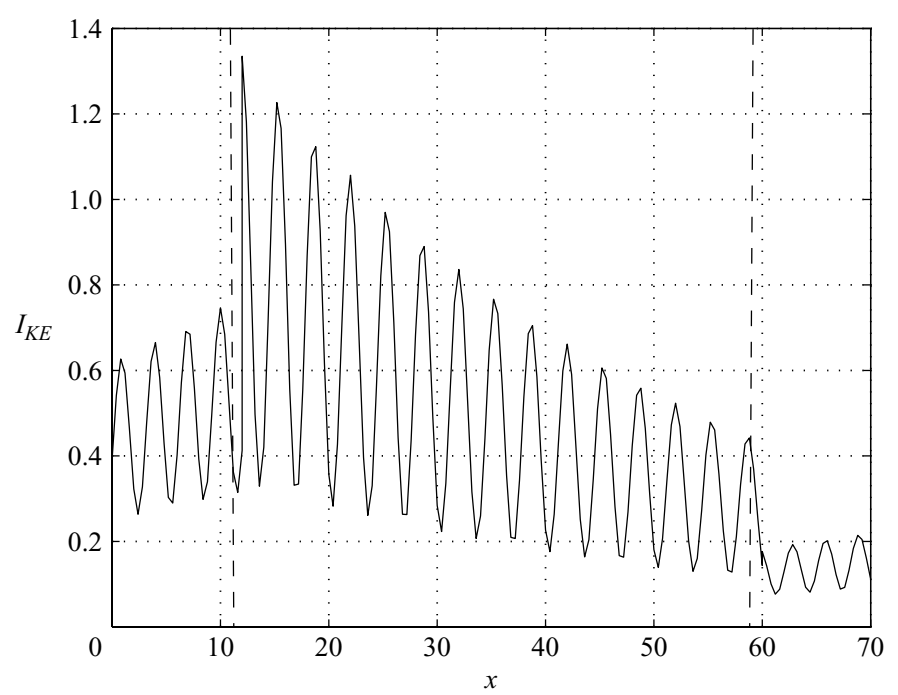

FIGURE 13. Streamwise variation of the perturbation enstrophy integral for the same case as in figure 10. Other features as in figure 10.

numerical simulations of Davies \& Carpenter (1997). It can be seen that there is close agreement between the present theory and the results of Davies and Carpenter. The slight mismatch at the leading and trailing edges that is evident in figure 10 is because evanescent modes have not been used for smoothening the edge results. Without this smoothening process one can clearly see that the jumps are the same irrespective of whether hinged-joint or clamped-joint edge conditions are used. This is also fully confirmed by the numerical simulations. More discussions on this point have been given earlier, at the end of $\S 4.6$. The jump in vorticity can be clearly seen in figure 11 . The jumps at the junctions are even more clearly seen in the plots of the enstrophy and kinetic energy integrals given respectively in figures 12 and 13 . These agree well 
with their counterparts obtained by numerical simulation and given in figure $16(a, b)$ of Davies \& Carpenter (1997).

The jumps in the various flow quantities that exist at the junctions follow readily from our theory. It is not so straightforward to determine whether a particular jump is upwards or downwards. The only location where the jump in the streamfunction can be determined readily with absolute certainty is at the centreline where the normalized eigenfunctions upstream and downstream of the junction are equal. At other values of $y$ the upstream and downstream eigenfunctions differ in value, as can be seen from figure 6 . Nevertheless, some idea may be obtained about the jumps by studying the eigenfunctions and vorticity functions respectively in figures 6 and 7 . For instance, let us assume that the jump in amplitude $\lambda$, strictly speaking the ratio of the values of the respective streamfunctions, viz. TSr and TSc, at the channel centreline is $\lambda=1$. The kinetic energy integral is dominated by the wall value of $\phi^{\prime}$. This is zero for TSr but non-zero for TSc. Hence the jump in the kinetic energy integral is upwards. On the other hand the vorticity integral is dominated by the wall value of $\phi^{\prime \prime}$. This is large for the TSr and somewhat less for the TSc. Hence the jump in the vorticity integral from TSr to TSc is downwards. These trends continue despite $\lambda$ being greater than 1 , i.e. $\lambda \simeq 1.2$.

\subsection{Results for TS frequency above cutoff}

Next we consider the above cutoff case. There is a plethora of propagating eigenmodes over the compliant panel. Despite this, we are able to compare the results based on the present theory with those based on numerical simulations using the methods in Davies \& Carpenter (1997). Some of these results already exist in Davies and Carpenter. Some new results were also generated. Therefore, for purposes of comparison we consider the case where the compliant panels have the same properties as in Davies and Carpenter (1997), i.e. $R e=12000$ and $\bar{\omega}=0.24$ and wall parameters

$$
m=\frac{1}{3}, \quad d=0, \quad K=1.92 \times 10^{7}, \quad B=4 K .
$$

We consider two panel lengths for the compliant panel. The first is $100 \mathrm{~h}$ (see figure 1 for $h$, which is the half-width of the channel). This is the same as for all the simulations described in Davies \& Carpenter (1997). We also consider a long panel of length $340 h$. For this long panel the TSc wave, generated at the leading-edge junction and which travels and decays downstream, has a negligible value by the time it reaches the trailing-edge junction. Also the $\mathrm{V}$ wave, generated at the trailing-edge junction and which travels and decays upstream, has a negligible value by the time it reaches the leading-edge junction. In the subsequent discussions these two panels will be referred to respectively as the 'short panel' and the 'long panel'.

First we consider the short panel. The wall displacement $\eta$ for each of the component waves in the compliant section are shown in figure 14 based on the present theory. The results compare very well with the corresponding figure $17(a-d)$ in Davies \& Carpenter (1997). Figure 15 is very interesting. It shows plots of the composite wall vorticity $\zeta_{w}$ for the entire panel, viz. rigid-compliant-rigid, obtained both by the present theory and also generated by numerical simulation using the methods in Davies and Carpenter (1997). Apart from very good general overall agreement, the two figures, respectively by the present theory and by numerical simulations, show a very interesting feature. When the compliant panel length is increased slightly, there is a distinct change in the amplitude of the TSr wave downstream of the trailing-edge junction. This is because, as described in the theory in $\S 4.5$ earlier, there are actually 


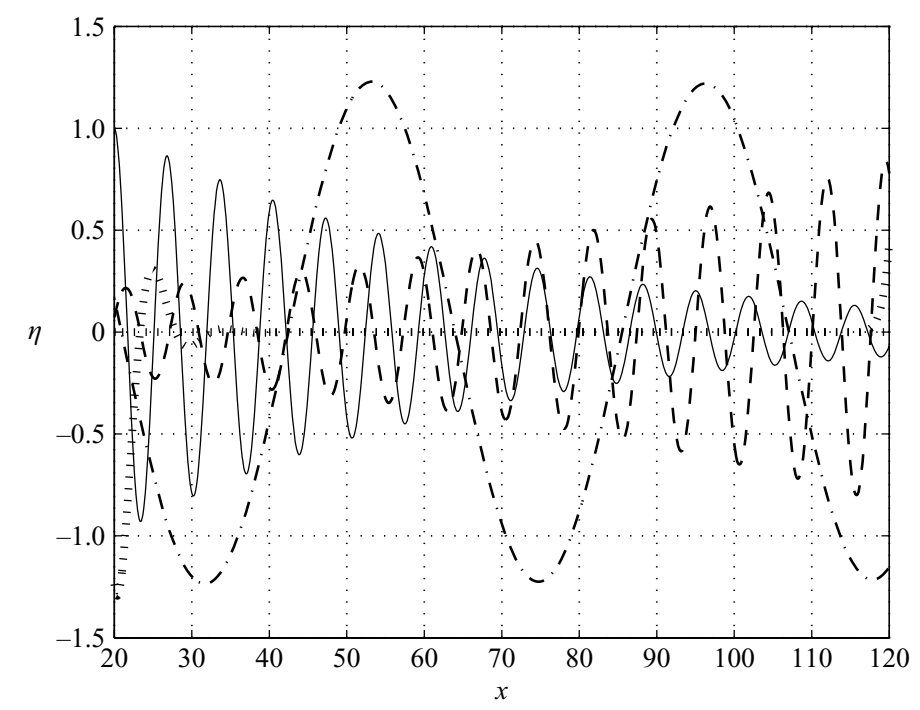

FIGURE 14. Wall displacements for the various modes above cutoff. Solid line $(-)$ is for the TS wave, broken line (- - ) is for the V wave, dash-dot line $(-\cdot-)$ is for the $\mathrm{L}$ wave and the broken ladder-line $(. .$.$) , at the two edges, are for the evanescent modes. Compare with$ figures $(17 a, b, c, d)$ in Davies \& Carpenter (1997).

two wave drivers present at the trailing-edge junction. The first is the TSc-TSr wave driver and the second is the $\mathrm{V}-\mathrm{TSr}$ wave driver. The latter forms because the real part of the pseudo group velocity $c_{p}$ of the $\mathrm{V}$ wave is positive. The change in amplitude of the downstream TSr wave occurs because the slight increase in panel length causes a phase change between the TSc-TSr wave driver and the $\mathrm{V}-\mathrm{TSr}$ wave driver. The resulting amplitude of the TSr wave, which is due to the superposition of the respective contributions due to the two wave drivers, changes with the relative change in phase difference induced by the slight change in panel length. The agreement between the two figures in figure 15 clearly vindicates the theory, the concept as well as the existence of the pseudo group velocity. This also gives a new mechanism of wave energy transfer across two different wave-bearing media.

Next we consider the long panel. Figure 16 is similar to figure 15 for the short panel, and shows the composite-wall vorticity $\zeta_{w}$ for the entire panel, viz. rigidcompliant-rigid, obtained both by the present theory and also generated by numerical simulation using the methods in Davies \& Carpenter (1997). There is again very good general overall agreement between theory and numerical simulations. In this figure also the compliant panel length is changed slightly, but this time the envelope of the TSr wave, downstream of the trailing-edge junction, does not change in amplitude in either of the two figures corresponding respectively to present theory and numerical simulations. This is because the TSc wave, generated at the leading edge and propagating and decaying downstream, becomes negligible when it reaches the trailing-edge junction. This is further illustrated in figure 17 where only the TSc wave is shown in the compliant segment. It is interesting to see in figure 17 the emergence of the downstream TSr wave despite the compliant-side TSc wave having become negligible at the trailing-edge junction. Figure 18 shows only the 

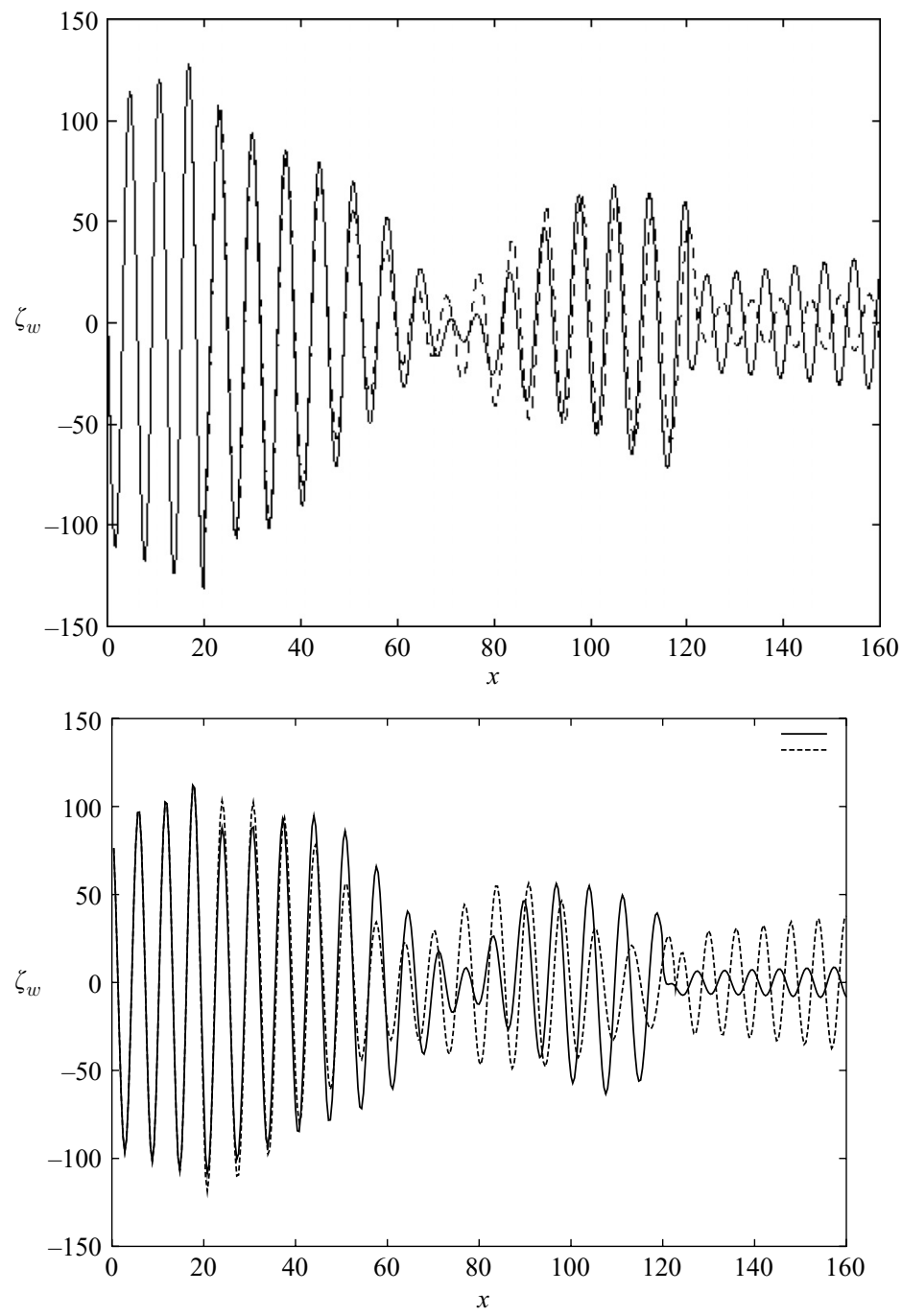

FIGURE 15 . Wall vorticity $\zeta_{w}$ for the entire panel, rigid-compliant-rigid, with a short compliant panel $(100 h)$, for the case above cutoff. Note the change in amplitude in the downstream rigid-wall vorticity, with slight change in length of the compliant panel. The leading and trailing edges of the panel are at $x=\ell_{1}=20$ and $x=\ell_{2}=120$ (and 120+) respectively. Upper figure is by present theory and lower figure is by numerical simulations using the methods of Davies \& Carpenter (1997). For the upper figure broken line is for slightly longer panel and solid line is for slightly shorter panel. For the lower figure it is the other way round. Figure is indicative of the existence of two wave drivers at the trailing-edge junction, viz. TSc-TSr and $\mathrm{V}-\mathrm{TSr}$.

$\mathrm{V}$ wave in the compliant segment. It is clear from this figure that only the $\mathrm{V}-$ TSr wave driver creates the downstream rigid-side TSr wave. Since this is the only wave driver existing, a slight change in the compliant panel length does not cause any change in the amplitude of the downstream TSr wave. Again this figure also vindicates the existence of the V-TSr wave driver as well as that of the pseudo group velocity and a new mechanism of wave energy transfer across different wave-bearing media. 

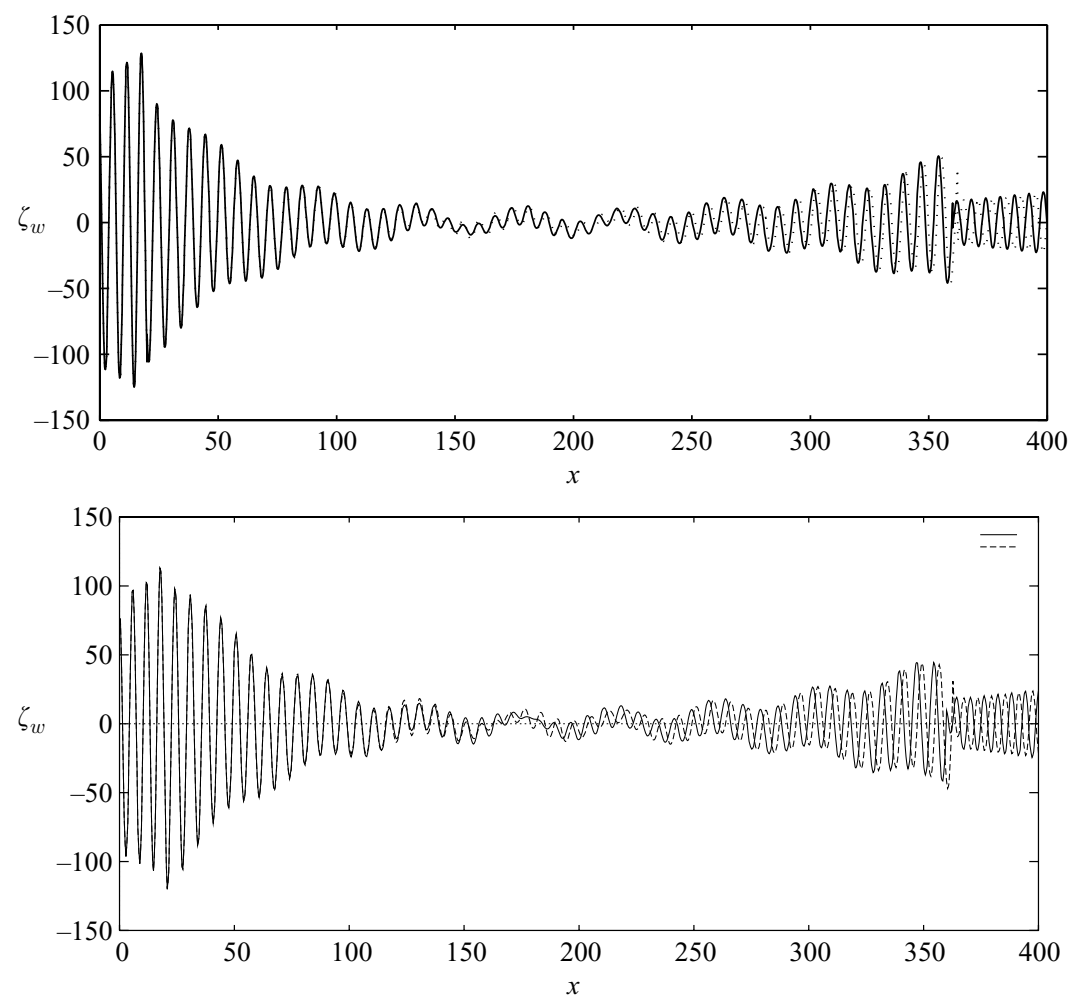

FIGURE 16. Wall vorticity $\zeta_{w}$ for the entire panel, rigid-compliant-rigid, with a long compliant panel (340h), for the case above cutoff. Note there is no change in amplitude in the downstream rigid-wall vorticity, with slight change in length of the compliant panel. The leading and trailing edges of the panel are at $x=\ell_{1}=20$ and $x=\ell_{2}=360$ (and 360+) respectively. Upper figure is by present theory and lower figure is by numerical simulations using the methods of Davies \& Carpenter (1997).

Finally we look at the rest of table 3 for the above cutoff cases. The jump for the TSr-TSc pair at the leading-edge junction, where the regular group velocity $c_{g}$ is used in the theoretical calculations, is well confirmed by the numerical simulation results. It is difficult to do this comparison at the trailing-edge junction for the TSc-TSr pair because for the short panel there are two wave drivers present, viz. TSc-TSr and V-TSr, at the trailing-edge junction. It is quite tedious to filter out the individual contributions of each wave driver from the downstream TSr wave in the numerical simulations. However, as shown in table 3 , the V-TSr jump is well confirmed by theory and numerical simulations, since the long panel simulation results are used where there is only one wave driver, viz. V-TSr, at the trailing-edge junction.

Table 3 also shows the jump results for the L wave at the leading- and trailing-edge junctions. The 'theoretical results' are only a blind application of the formulae for the jumps. The results between theory and numerical simulations show no agreement whatsoever, vindicating our earlier contention that the L wave, being nearly nonvortical, is not a candidate for the wave driver theory. 


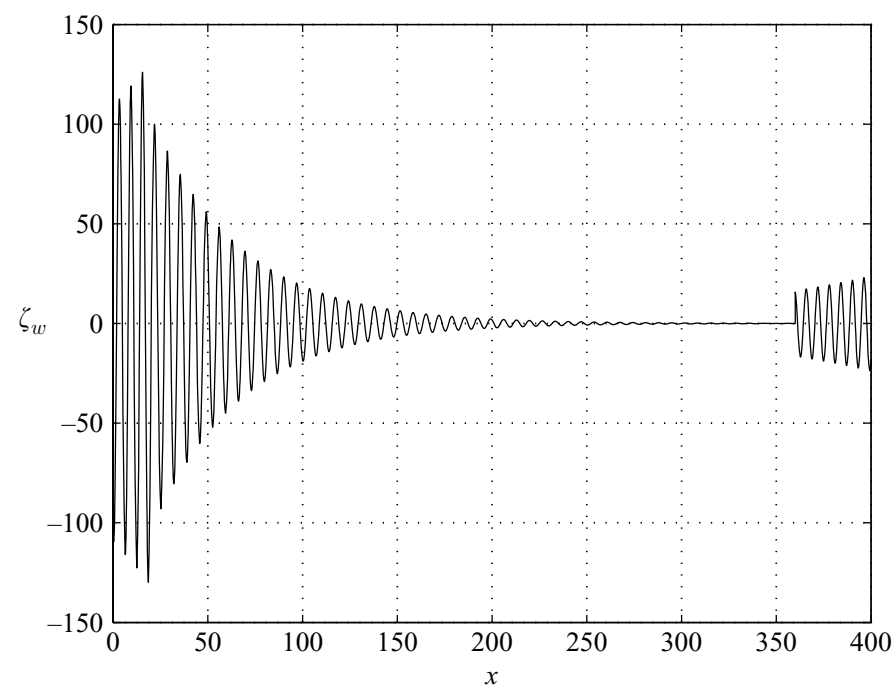

FIGURE 17. Same data as in figure 16, showing only the TS wave in the compliant panel. Note that the TS wave has decayed completely by the time it reaches the trailing-edge junction. Yet, there is a rigid-side TS wave downstream of the trailing edge junction.

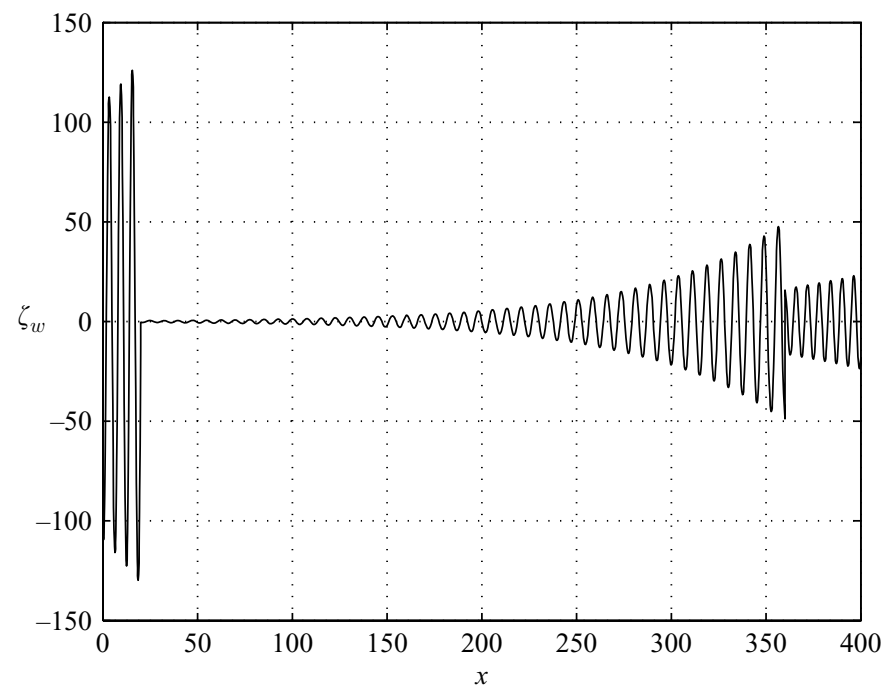

FIGURE 18. Same data as in figure 16, showing only the V wave in the compliant panel. Note that the $\mathrm{V}$ wave has decayed completely by the time it reaches the leading-edge junction. The rigid-side TS wave, downstream of the trailing edge junction, is obviously due to the wave driver created by the $\mathrm{V}$ wave.

\section{Conclusions}

We have presented a novel method for analysing the behaviour of vortical waves incident on junctions between two different wave-bearing media. There is a spatially homogeneous eigenproblem corresponding to each wave-bearing medium. It is assumed that the far fields away from the immediate vicinity of the junction correspond to superpositions of one or more (homogeneous) eigenstates. Typically 
the upstream state corresponds to the incident wave plus, in some cases, a reflected wave, while the downstream state corresponds to the transmitted wave plus other waves excited by the interaction of the incident wave and the wall to match the edge conditions at the junctions. It is also assumed that there is a rapid change in the neighbourhood of the junction between superpositions of upstream eigenstates and downstream eigenstates so that the junction region is asymptotically narrow. Given this scenario, the key question became how to determine the ratios of the amplitudes of the downstream eigenstates to the amplitude of the incident wave. We showed that the junction is equivalent to a virtual wave driver. Based on this analogy, for a particular problem we showed how the amplitudes of the downstream transmitted eigenfunction can be determined in terms of the amplitude of the incident wave.

We believe that aspects of this method have general applicability, but it is applied here to a specific problem, namely, the behaviour of a TS wave propagating in plane Poiseuille flow and incident on a junction between rigid and compliant walls. More specifically we considered the problem investigated by Davies \& Carpenter (1997) using direct numerical simulations. In this case there is a finite compliant panel having junctions with rigid walls at both the leading and trailing edges of the panel. Thus for the leading-edge junction the incident wave corresponds to a single rigid-wall TS wave; theoretically there may be one or more eigenstates downstream of the junction, but it was found that only the compliant TS wave emerges from the leading-edge junction, both for below and above cutoff cases. For the trailing-edge junction, on the other hand, there may be more than one eigenstate upstream, but there is only one transmitted eigenstate downstream, namely a rigid-wall TS wave.

We carried out HFLT upstream and downstream of the junction of the linearized vorticity transport equation (derived from the Navier-Stokes equations). In this way we derived the governing equations and boundary conditions for the problem and showed that at the junction a discrete structure could be educed from the near field, which we called the virtual wave driver. This wave driver is strongly vortical in nature.

In order to provide a simple illustration of our concepts and methods, we first considered the problem of a known wave driver located at streamwise location $x=0$ in a rigid-wall plane Poiseuille flow. We expanded our wave driver function in terms of $\bar{g}_{k}=-\mathrm{i}\left(\bar{\phi}_{k}^{\prime \prime}-\bar{\alpha}^{2} \bar{\phi}_{k}\right)(k=1,2,3, \ldots, \infty)$, where $\alpha$ and $\bar{\phi}_{k}$ are respectively the downstream far-field complex wavenumber (eigenvalue) and corresponding higherorder eigenfunctions. By using the orthogonality relationship between the $\bar{g}_{k}$ and the higher-order adjoint eigenfunctions $\bar{\theta}_{k}$ we were able to determine the amplitude of the downstream far-field eigenfunction solely in terms of the driver function at $x=0$. Comparisons were made with the results of a numerical simulation where the driver took the form of a harmonically oscillating wall-normal velocity with a narrow Gaussian distribution in $x$ centred at $x=0$ applied at the wall. The theoretical predictions agreed reasonably well with the results of the numerical simulation.

We then turned to the main problem where a TS wave is incident on a junction between a rigid and compliant wall and creates a virtual wave driver that generates a downstream transmitted TS wave. The wave driver is like a 'half source cum half sink'. It is a 'sink' for the incoming wave and a 'source' for the outgoing wave. The basic inhomogeneous differential equations in Fourier-Laplace space was solved by considering generalized eigenstates in $\omega_{k}$ in the Fourier-Laplace domain and after giving due attention to the wall boundary conditions, particularly for the compliant cases. For the compliant cases, two routes were found by which the singularity for $\alpha \rightarrow \bar{\alpha}$ could be approached in Fourier-Laplace space. One route was appropriate for the 'source' side of the wave driver, and the other was appropriate for the 'sink' 
side of the wave driver. The latter also gave a new group velocity, called the pseudo group velocity herein, which constitutes a new mechanism of wave energy transfer across two different wave-bearing media.

It was also seen that the long wave, which is nearly non-vortical, is neither generated from a wave driver nor does it create a wave driver. In fact, the long wave and the compliant vortical wave, which are both wall-based waves, are created at the edges of the junctions from the matching of the edge conditions. The wave driver, being strongly vortical in nature, can be created by vortical waves only, and only vortical waves can emerge from a wave driver.

The main conclusions are now summarized as follows:

(i) When a vortical wave is incident on a junction the amplitudes of the far-field transmitted and reflected waves can be predicted solely in terms of the amplitude of the incident wave, given a knowledge of all the far-field eigenstates involved.

(ii) The interaction of the incident wave with the junction is equivalent to the creation of a virtual wave driver.

(iii) Each type of incident wave creates its own virtual wave driver, when the circumstances are conducive for the creation of a wave driver.

(iv) A novel form of group velocity, the pseudo group velocity, has been derived for the waves over the compliant panel. This is used for the 'sink' side of the wave driver. The pseudo group velocity also typifies a new mechanism of wave energy transfer across two different wave-bearing media.

(v) When the wave frequency is below the cutoff frequency of the compliant panel, the jumps in wave amplitude at the leading- and trailing-edge junctions are nearly reciprocal. The lack of complete reciprocity is due to the use of regular group velocity at the leading-edge junction whereas the pseudo group velocity is used at the trailingedge junction. In the below cutoff case the values of the two types of group velocity differ only slightly. But they differ considerably in the above cutoff case for which the jumps in wave amplitude are much greater at the leading-edge junction than at the trailing-edge junction.

(vi) For very long compliant panels the TS wave can decay to virtually zero amplitude by the time it reaches the trailing-edge junction. Nevertheless, a TS wave of non-negligible amplitude is still created at the trailing-edge junction and propagates downstream along the rigid wall outlet section. This downstream wave is generated by a virtual wave driver created by the vortical $\mathrm{V}$ wave at the trailing edge.

The work described in this paper was carried out in part with the support of the Engineering and Physical Sciences Research Council, UK, which made it possible for Professor Sen to make extended visits to the University of Warwick through visiting research fellowship grants, and also for Professor Carpenter to make two-week visits to the Indian Institute of Technology, Delhi, on a good few occasions.

\section{Appendix. The adjoint eigensystem}

The Orr-Sommerfeld equation, $L(\alpha) \phi=0$, where the operator $L(\alpha)$ is defined in (2.17), can be written in the form

$$
L(\alpha)\left(\equiv \sum_{n=0}^{4} A_{n}(y) D^{n} \phi\right)=0,
$$


where $D^{n}=1$ when $n=0$ and $d^{n} / d y^{n}$ otherwise; the coefficients are defined as follows: $A_{0}=\alpha^{4}+\mathrm{i} \alpha^{3} \operatorname{Re}(\bar{u}-c)+\mathrm{i} \alpha \operatorname{Re} \bar{u}^{\prime \prime}, A_{1}=0, A_{2}=-2 \alpha^{2}-\mathrm{i} \alpha \operatorname{Re}(\bar{u}-c), A_{3}=0, A_{4}=1$,

and $c=\omega / \alpha$. By definition the adjoint equation is defined as

$$
\bar{L}(\alpha) \theta\left(\equiv \sum_{n=0}^{4}(-1)^{n} D^{n}\left[A_{n}(y) \theta\right]\right)=0,
$$

where $\theta$ is the adjoint eigenfunction. By carrying out the operations indicated in (A 3), we obtain the adjoint equation explicitly as

$$
\theta^{\prime \prime \prime \prime}+\left[-2 \alpha^{2}-\mathrm{i} \alpha \operatorname{Re}(\bar{u}-c)\right] \theta^{\prime \prime}-\mathrm{i} \alpha \operatorname{Re} \bar{u}^{\prime} \theta^{\prime}+\left[\alpha^{4}+\mathrm{i} \alpha^{3} \operatorname{Re}(\bar{u}-c)\right] \theta=0 .
$$

The bilinear concomitant is defined by the following relationship:

$$
B L C=\int_{0}^{1}[L \phi] \theta \mathrm{d} y-\int_{0}^{1}[\bar{L} \theta] \phi \mathrm{d} y .
$$

By applying partial integration and using (A 1) and (A 3) the following explicit form of the bilinear concomitant can be derived:

$$
\begin{aligned}
B L C=\left[\theta \phi^{\prime \prime \prime}-\theta^{\prime} \phi^{\prime \prime}+\theta^{\prime \prime} \phi^{\prime}-\theta^{\prime \prime \prime} \phi+\left(-2 \alpha^{2}+\mathrm{i} \alpha \operatorname{Rec}\right)\left(\theta \phi^{\prime}-\theta^{\prime} \phi\right)\right]_{0}^{1} \\
+\left[-\mathrm{i} \alpha \operatorname{Re}\left(\theta \bar{u} \phi^{\prime}-\theta^{\prime} \bar{u} \phi-\theta \bar{u}^{\prime} \phi\right]_{0}^{1} .\right.
\end{aligned}
$$

The bilinear concomitant is used to determine the boundary conditions for the adjoint eigensystem by setting it equal to zero at the wall $(y=0)$ and channel centreline $(y=1)$. In this way we readily find

$$
\theta^{\prime}=\theta^{\prime \prime \prime}=0 \quad \text { at } y=1 \text {. }
$$

At $y=1$, the boundary conditions for $\theta$ are the same as for $\phi$, Also, at the wall, $y=0$, the boundary conditions for $\theta$, respectively for rigid and compliant walls, are given as follows:

$$
\text { Rigid: } \quad \theta=0, \quad \theta^{\prime}=0, \quad \text { at } y=0,
$$

$$
\text { Compliant: } \quad \theta^{\prime}=0, \quad \bar{B} \theta^{\prime \prime \prime}+\theta^{\prime \prime}+S \theta=0, \quad \text { at } y=0,
$$

where in $(\mathrm{A} 9 a)$,

$$
S=\left(\bar{B} / \zeta-2 \alpha^{2}\right), \quad \bar{B}=c / \bar{u}^{\prime}, \quad \zeta=\frac{Y_{0}}{\left[Y_{0} \frac{\alpha^{2}}{\bar{B}}+\alpha^{2} R e\right]},
$$

and

$$
Y_{0}=-\frac{\mathrm{i} c}{m \alpha\left(\bar{c}_{0}^{2}-c^{2}-\mathrm{i} c d / \alpha\right)}, \quad \bar{c}_{0}^{2}=B \alpha^{2} / m+K /\left(m \alpha^{2}\right) .
$$

\section{REFERENCES}

Ashris, D. E. \& Reshotko, E. 1990 The vibrating ribbon problem revisited. J. Fluid Mech. 213, 531-547.

Billingham, J. \& King, A. C. 2000 Wave Motion. Cambridge University Press.

CARpenter, P. W. \& Garrad, A. D. 1985 The hydrodynamic stability of flow over Kramer-type compliant surfaces. Part 1. Tollmien-Schlichting instabilities. J. Fluid Mech. 155, 465-510. 
CARPENTER, P. W. \& Morris, P. J. 1990 The effect of anisotropic wall compliance on boundary-layer stability and transition. J. Fluid Mech. 218, 171-223.

Carpenter, P. W. \& Sen, P. K. 2003 Propagation of waves across junctions between rigid and flexible walls. In Flow Past Highly Compliant Boundaries and in Collapsible Tubes (ed. P. W. Carpenter \& T. J. Pedley), pp. 147-166. Kluwer.

Carpenter, P. W., Sen, P. K., Hegde, S. \& Davies, C. 2002 Wave propagation in flows across junctions between rigid and flexible walls. ASME Paper IMECE 2002-32202.

DAVIES, C. \& CARPENTER, P. W. 1997 Numerical simulation of the evolution of Tollmien-Schlichting waves over finite compliant panels. J. Fluid Mech. 335, 361-392.

Drazin, P. G. \& ReId, W. H. 1981 Hydrodynamic Stability. Cambridge University Press.

GASTER, M. 1965 On the generation of spatially growing waves in a boundary layer. J. Fluid Mech. 22, 433-441.

Grotberg, J. B. \& Jensen, O. E. 2004 Biofluid mechanics in flexible tubes. Annu. Rev. Fluid Mech. 36, 121-147.

HegDe, S. 2002 Study of small disturbance waves across alternate rigid and compliant panels, with analytical jump conditions at the junctions. PhD thesis, Indian Institute of Technology, Delhi.

Heil, M. \& Jensen, O. E. 2003 Flow in deformable tubes and channels. In Flow Past Highly Compliant Boundaries and in Collapsible Tubes (ed. P. W. Carpenter \& T. J. Pedley), pp. 15-49, Kluwer.

Henningson, D. S. \& Schmid, P. J. 1992 Vector eigenfunction-expansions for plane channel flows. Stud. Appl. Math. 87, 15-43.

HiLl, D. C. 1995 Adjoint systems and their role in receptivity problem for boundary layers. J. Fluid Mech. 292, 183-204.

Howe, M. S. 1998 Acoustics of Fluid-Structure Interactions. Cambridge University Press.

INCE, E. L. 1926 Ordinary Differential Equations. Longmans, Green and Co.

Kramer, M. O. 1960 Boundary-layer stabilization by distributed damping. J. Am. Soc. Nav. Engrs. 72, 25-33.

LANDAHL, M. T. 1962 On the stability of a laminar incompressible boundary layer over a flexible surface. J. Fluid Mech. 13, 609-632.

Lighthill, J. 1978 Waves in Fluids. Cambridge University Press.

Lucey, A. D., Sen, P. K. \& Carpenter, P. W. 2003 Excitation and evolution of waves on an inhomogeneous flexible surface in a mean flow. J. Fluids Struct. 18, 251-267.

Luo, X. Y. \& Pedley, T. J. 1995 A numerical simulation of steady flow in a 2-D collapsible channel. J. Fluids Struct. 9, 149-174.

Luo, X. Y. \& Pedley, T. J. 1996 A numerical simulation of unsteady flow in a 2-D collapsible channel. J. Fluid Mech. 314, 191-225.

Manuilovich, S. V. 1992 Passage of an instability wave through a channel section of variable width (in Russian). Izv. Ros. Akad Nauk, Mekh. Zhidk. Gaza, No. 2, 34-41 (Translation in Fluid Dyn. 27, 177-182).

Manuilovich, S. V. 2001 Propagation of Tollmien-Schlichting wave in a boundary layer over flexible path of a wall. In book of abstracts for IUTAM Symposium on Flow in Collapsible Tubes and Past Other Highly Compliant Boundaries, University of Warwick.

Manuilovich, S. V. 2003 Propagation of perturbations in plane Poiseuille flow between walls of nonuniform compliance. Fluid Dyn. 38, 529-544.

Manuilovich, S. V. 2004a Transformation of the instability wave under an abrupt change. Dokl. Phys. 49, 342-346.

ManulLovich, S. V. $2004 b$ Propagation of Tollmien-Schlichting waves over the junction between stiff and flexible panels (in Russian). Mekh. Zhidk. i Gaza, No. 5, 31-48.

Nguyen, V. B., Païdoussis, M. P. \& Misra, A. K. 1994 A CFD-based model for the study of the stability of cantilevered coaxial cylindrical shells. J. Sound Vib. 176, 105-125.

Noble, B. 1958 Methods Based on the Wiener-Hopf Technique. Pergamon Press.

PAïDoussis, M. P. 1998 Fluid-Structure Interactions: Slender Structures and Axial Flow. vol. 1, Academic Press.

Pedrizzetti, G. 1998 Fluid flow in a tube with an elastic membrane insertion. J. Fluid Mech. 375, 39-64.

Schmid, P. J. \& Henningson, D. S. 2001 Stability and Transtion in Shear Flows. Springer. 
Sen, P. K. \& Arora, D. S. 1988 On the stability of laminar boundary layer flow over a flat plate with a compliant surface. J. Fluid Mech. 197, 201-240.

Sen, P. K., Hegde, S. \& Carpenter, P. W. 2002 Simulation of small disturbance waves over alternate rigid and compliant panels. Indian J. Engng Mater. Sci. 9, 409-413.

Sen, P. K., Hegde, S. \& Carpenter, P. W. 2003 Propagation of small disturbance waves in a fluid flow across junctions between rigid and compliant panels. Def. Sci. J. 53, 189-198.

WiPLIER, O. \& EhrEnstein, U. 2000 Numerical simulation of linear and nonlinear disturbance evolution in a boundary layer with compliant walls. J. Fluids Struct. 14, 157-182. 